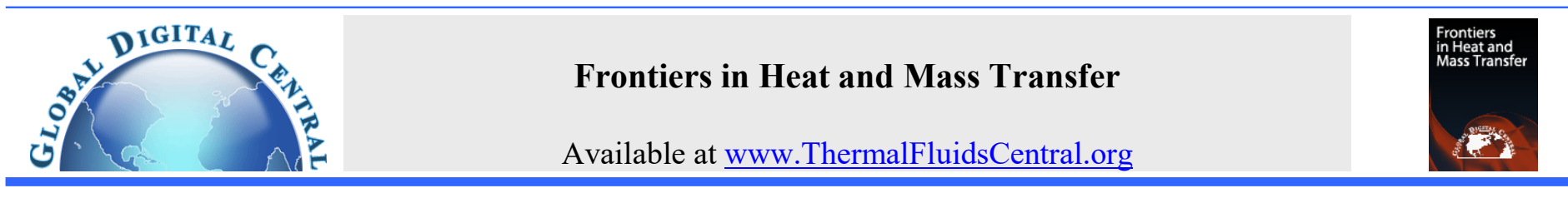

\title{
NUMERICAL SIMULATION OF SLIP INFLUENCE ON ELECTRIC CONDUCTING VISCOELASTIC FLUID PAST AN ISOTHERMAL CYLINDER
}

\author{
CH. Amanulla ${ }^{a, b}$, N. Nagendra ${ }^{b}$, M. Suryanarayana Reddy ${ }^{c}$ \\ a Department of Mathematics, Jawaharlal Nehru Technological University, Anantapur, Anantapuramu-515002, India. \\ ${ }^{b}$ Department of Mathematics, Madanapalle Institute of Technology and Science, Madanapalle-517325, India. \\ ${ }^{c}$ Department of Mathematics, JNTUA College of Engineering, Pulivendula-516390, Andhra Pradesh, India.
}

\begin{abstract}
The present study deals with the computational analysis on an electrically conducting magneto viscoelastic fluid over a circular cylinder. Prescribed partial slip effects are also taken into account. The governing physical problem is tackled numerically by using the highly efficient and reliable Keller box algorithm. Impact of sundry physical parameters on physical quantities of interest are evaluated. The influence of Williamson viscoelastic fluid parameter, magnetic body force parameter, Thermal and velocity (hydrodynamic) slip parameters, stream wise variable and Prandtl number on thermos-fluid characteristics are studied graphically. The model is relevant to the simulation of magnetic polymer materials processing.

Keywords: Velocity and Thermal slip; Magneto-hydrodynamics; Circular Cylinder; Williamson fluid parameter; Viscoelastic model.
\end{abstract}

\section{INTRODUCTION}

Recent era is transferring of heat through lubricants due to circular cylinder plays major role in the extrusion process of fibre technology, making of polymer sheets and plastic films, manufacturing paper, glass blowing, spinning of metals and plastic films, etc. Hunegnaw and kishan (2014) discussed the effects of variable viscosity on MHD flow past a stretching sheet in porous medium. Over an exponentially stretching sheet, the slip effects on MHD flow is analyzed by Hayat et al. (2016) Swati and Anuar (2012) explained in detail regarding the Convection flow past stretching cylinder. Rasekh (2012) analyzed the heat source effect due to a stretching circular cylinder. Salahuddin et al. (2016) investigated by Keller box method on MHD Pseudo-plastic flow past a stretching cylinder. Williamson (1929) first studied the flow of pseudo plastic materials. The Williamson fluid model tends to a Newtonian fluid at a very high wall shear stress i.e. when the wall stress is far greater than yield stress. To improve processing of many types of polymers, numerous investigators have conducted simulations of Williamson flow dynamics using many computational and analytical methods. These studies have included heat transfer (important for thermal treatment), mass transfer (critical to doping modification of polymers), viscous heating, Magnetohydrodynamic (for electroconductive polymers) and many other phenomena. Salleh et al. (2011) used the Crank-Nicolson method to analyze flow from a circular cylinder with Newtonian heating. Hayat et al. (2016) used Homotopy analysis method (HAM) to simulate the MHD flow of Powell-Eyring fluid by a stretching cylinder. Makanda et al. (2015) analyzed the radiative heat flux effect on hydromagnetic dissipative Casson slip fluid flow from a horizontal circular cylinder in porous media. Rao and Sekhar (2000) investigated MHD flow past a circular cylinder with applied magnetic field. Grigoriadis et al. (2010) analyzed the MHD flow past a circular cylinder using the immersed boundary method. These studies however did not consider the Williamson model. This is a shear thinning non-Newtonian model which quite accurately simulates polymer viscoelastic flows over a wide spectrum of shear rates. In Williamson fluids the viscosity is reduced with rising shear stress rates. This model has found some popularity in engineering simulations.

Magnetic field effects on a conducting fluid received good attention from researchers. This is because hydromagnetic flow and heat transfer have become more important in industrially. For example, many metallurgical processes such as drawing, annealing and tinning of copper wires involve cooling of continuous strips or filaments by drawing them through a quiescent fluid. Controlling the rate of cooling in these processes can affect the properties of the final product. This can be done by using an electrically-conducting fluid and applying a magnetic field. The similar solutions to study the effects of mass transfer (suction/injection) and chemical reaction on the steady mixed convection boundary-layer flow over a rotating cone were presented by Saleem et al. (2016). Mahapatra et al. (2014) analyzed the natural convection upon a horizontal flat plate with magnetic effects. The influence of heat absorption and source on MHD past stretching sheet in a non-Darcian medium was examined by Ibrahim and Shankar (2016). Amanulla et al. (2018) analyzed a boundary layer in natural convection in the presence of MHD convective heating and thermal radiation effects on Williamson fluid flow past a vertical plate using the finite difference method.

The previous studies invariably assumed the classical "no-slip" condition at the boundary. Slip effects have however shown to be important in numerous polymeric transport processes including the production stage of polymers from the raw (monomeric) materials and in converting high-molecular-weight products into specific products by W.B. Black (2000). Many researchers, primarily in chemical engineering have therefore studied, both experimentally and numerically, the influence of wall slip on polymer dynamics. Important works in this regard include Wang et al. (1996) who considered low density polyethylene liquids, Piau et al. (1995) who addressed polymer extrudates, Piau and Kissi (1994) who quantified macroscopic wall slip in polymer melts, Lim and Schowalter (1989) who studied boundary

\footnotetext{
${ }^{*}$ Corresponding author.Email: amanullamsc@gmail.com
} 
slip in polybutadiene flows and Hatzikiriakos and Kalogerakis (1994) who also studied molten polymer wall slip. Wall slip in thermal polymer processing was considered by Liu and Gehde (2016) in which slip was shown to significantly modify temperature distribution in polymers. Saleem and Nadeem (2015) used optimal Homotopy analysis method (OHAM) for nonlinear boundary layer flow from a rotating cone with presence of slip and viscous dissipation effects. Amanulla et al. (2017) carried out a heat slip flow analysis on the basis of a mathematical model managed by the boundary layer hypotheses while using the non-similarity method to reduce the governing equations. Hatzikiriakos and Mitsoulis (2009) presented closed-form solutions and finite element computations for wall slip effects on pressure drop of power-law fluids in tapered dies. Many studies of both momentum (hydrodynamic or velocity) slip and thermal slip on transport phenomena have also been reported. A Computational analysis of the heat and mass transfer through a Nanofluid flow over the semi-infinite plate was established by Amanulla et al. (2018). Another study was carried out by Amanulla (2017) on boundary layers of a non-Newtonian Nanofluid subject to convective boundary conditions. A study was conducted by Amanulla et al. (2017) to analyze a boundary layer in Magnetohydrodynamic free convection while employing a nonsimilarity technique to which partial differential equations are reduced to ordinary differential equations. For some non-Newtonian fluids, such as polymer melts, it is nowadays consensually accepted the existence of slip velocity between the fluid and the solid wall (Kaoullas and Georgiou (2015), Chiu-On Ng (2016), Afonso et al. (2013), Philippou et al. (2017), Panaseti and Georgiou (2017)). Ferrás et al. $(2012,2013,2014,2017)$ gives a detailed overview of the viscoelastic fluid slip flows.

The main objective of the present investigation is to study the effect of velocity and thermal slip effects on natural convection flow of Williamson fluid past a circular cylinder with magnetic field. The method of solution involves non-similarity transformation which reduces the partial differential equations into a set of non-linear ordinary differential equations. These non-linear ordinary differential equations have been solved by applying Keller Box Method with help of finite difference technique. The velocity, temperature, skin friction and heat transfer profiles for different values of flow parameters are presented in the figures. It is observed from all figures that the boundary conditions are satisfied asymptotically in all the cases which support the accuracy of numerical results.

\section{MATHEMATICAL THERMO-VISCOELASTIC FLOW MODEL}

Consider a steady incompressible flow along a continuously circular cylinder. The fluid is electrically conducting, so that the magnetic field is applied transversely. The cylinder is completely immersed in thermally stratified saturated medium with a variable ambient temperature $T_{\infty}(\mathrm{x})$, where $T_{w}(\mathrm{x})>T_{\infty}(\mathrm{x})$. Fig. 1 below gives the brief description of flow considered.

$\operatorname{div} \mathrm{V}=0$,

$\rho \frac{\mathrm{dV}}{\mathrm{dt}}=\operatorname{divS}+\rho b$

Where $\rho$ is the density of the fluid, $\mathrm{V}$ is the velocity vector, $\mathrm{S}$ is the Cauchy stress tensor, $b$ represents the specific body force vector, and $\mathrm{d} / \mathrm{dt}$ represents the material time derivate. The constitutive equations of the Williamson fluid model $(2017,2016,2014,2013,2007)$ are given as:

$$
\mathrm{S}=-\mathrm{pI}+\tau
$$

$\tau=\left(\mu_{\infty}+\frac{\left(\mu_{0}-\mu_{\infty}\right)}{1-\Gamma \dot{\gamma}}\right) A_{1}$,
Here $\mathrm{p}$ is the pressure, I is the identity vector, $\tau$ is the extra stress tensor, $\mu_{0}$ are the limiting viscosities at zero and at infinite shear rate, $\Gamma$ is the time constant $(>0), \mathrm{A}_{1}$ is the first Rivlin-Erickson tensor and $\dot{\gamma}$ is defined as follows:

$\dot{\gamma}=\sqrt{\frac{1}{2} \pi}$

$\pi=\operatorname{trace}\left(A_{1}^{2}\right)$

Here we considered the case for which $\mu_{\infty}=0$ and $\Gamma \dot{\gamma}<1$. Thus eq.

(4) can be written as:

$\tau=\left(\frac{\mu_{0}}{1-\Gamma \dot{\gamma}}\right) A_{1}$

By using binomial expansion, we get:

$\tau=\mu_{0}(1+\Gamma \dot{\gamma}) A_{1}$

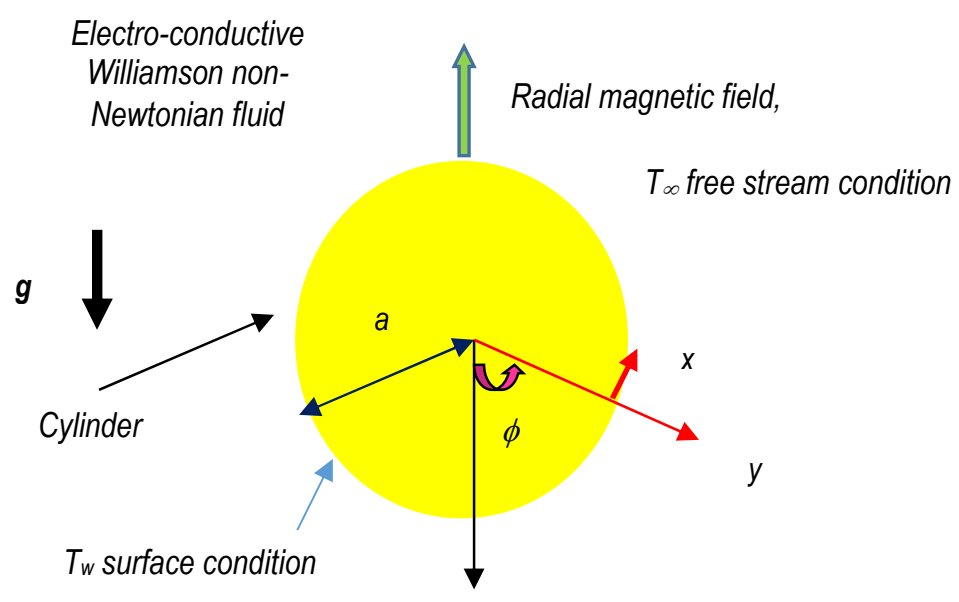

Fig. 1 Magnetohydrodynamic non-Newtonian heat transfer

The two-dimensional mass, momentum and energy boundary layer equations governing the flow in an $(\mathrm{x}, \mathrm{y})$ coordinate system may be shown to take the form:

$$
\frac{\partial u}{\partial x}+\frac{\partial v}{\partial y}=0
$$

$$
\begin{aligned}
u \frac{\partial u}{\partial x}+v \frac{\partial u}{\partial y}= & v \frac{\partial^{2} u}{\partial y^{2}}+g \beta\left(T-T_{\infty}\right) \sin \left(\frac{x}{a}\right) \\
& +\sqrt{2} v \Gamma \frac{\partial u}{\partial y} \frac{\partial^{2} u}{\partial y^{2}}-\frac{\sigma B_{0}^{2}}{\rho} u
\end{aligned}
$$

$u \frac{\partial T}{\partial x}+v \frac{\partial T}{\partial y}=\alpha \frac{\partial^{2} T}{\partial y^{2}}$

The boundary conditions for the velocity and temperature fields are

At $y=0, u=N_{0} \frac{\partial u}{\partial y}, v=0, T=T_{w}+K_{0} \frac{\partial T}{\partial y}$
As $y \rightarrow \infty, u \rightarrow 0, v \rightarrow 0, T \rightarrow T_{\infty}$ 
Here $N_{0}$ is the velocity slip factor, $K_{0}$ is the thermal slip factor and $T_{\infty}$ is the free stream temperature. For $N_{0}=0=K_{0}$, one can recover the no-slip case. The stream function $\psi$ is defined by $u=\frac{\partial \psi}{\partial y}$ and $v=-\frac{\partial \psi}{\partial x}$, and therefore, the continuity equation is automatically satisfied. In order to write the governing equations and the boundary conditions in dimensionless form, the following non-dimensional quantities are introduced:

$$
\begin{gathered}
\xi=\frac{x}{a}, \eta=\frac{y}{a} G r^{1 / 4}, f(\xi, \eta)=\frac{\psi}{v \xi G r^{1 / 4}}, \theta(\xi, \eta)=\frac{T-T_{\infty}}{T_{w}-T_{\infty}} \\
\operatorname{Pr}=\frac{v}{\alpha}, G r=\frac{g \beta\left(T_{w}-T_{\infty}\right) a^{3}}{v^{3}}, W e=\frac{\sqrt{2} v \Gamma x G r^{3 / 4}}{a^{3}}, M=\frac{\sigma B_{0}^{2} a^{2}}{\rho v \sqrt{G r}}
\end{gathered}
$$

The emerging momentum and heat (energy) conservation equations in dimensionless from assume the following form:

$$
\begin{aligned}
& f^{\prime \prime \prime}+f f^{\prime \prime}-f^{\prime^{2}}+W e f^{\prime \prime} f^{\prime \prime \prime}+\frac{\sin \xi}{\xi} \theta-M f^{\prime}=\xi\left(f^{\prime} \frac{\partial f^{\prime}}{\partial \xi}-f^{\prime \prime} \frac{\partial f}{\partial \xi}\right) \\
& \frac{1}{\operatorname{Pr}} \theta^{\prime \prime}+f \theta^{\prime}=\xi\left(f^{\prime} \frac{\partial \theta}{\partial \xi}-\theta^{\prime} \frac{\partial f}{\partial \xi}\right)
\end{aligned}
$$

The transformed dimensionless boundary conditions are reduced to:

At $\eta=0, f=0, f^{\prime}=S_{f} f^{\prime \prime}(0), \theta=1+S_{T} \theta^{\prime}(0)$

As $\eta \rightarrow \infty, f^{\prime} \rightarrow 0, \theta \rightarrow 0$

The skin-friction coefficient (surface shear stress) and the local Nusselt number (surface heat transfer rate) can be defined, respectively, using the transformations described above with the following expressions:

$$
\begin{aligned}
& G r^{-3 / 4} C_{f}=\xi f^{\prime \prime}(\xi, 0)+\frac{W e}{2} \xi\left(f^{\prime \prime}(\xi, 0)\right)^{2} \\
& G r^{-1 / 4} N u=-\theta^{\prime}(\xi, 0)
\end{aligned}
$$

All parameters are defined in the nomenclature.

\section{COMPUTATIONAL SOLUTION WITH KELLER BOX IMPLICT METHOD}

The transformed, nonlinear, multi-physical boundary value problem defined by Eqns. (14) -(16) can be solved via a number of numerical schemes. Here we implement a popular, second order accurate implicit finite difference method originally developed by Keller (1976). It has been used recently in MHD polymeric flow dynamics by $(2016,2017,2018)$ for viscoelastic models. The key stages involved are as follows:

a. Reduction of the Nth order partial differential equation system to $\mathrm{N}$ first order equations

b. Finite difference discretization

c. Quasilinearization of non-linear Keller algebraic equations

d. Block-tridiagonal elimination of linear Keller algebraic equations

In the Keller box scheme, the multi-degree, multi-order coupled partial differential equations defined in (14) and (15) are first reduced to a system of first order equations. These equations are then discretized with the finite difference approximations with appropriate step lengths in each coordinate direction. Introducing the new variables:

$f^{\prime}=u$ $u^{\prime}=v$

$\theta^{\prime}=t$

Eqns. (14) - (15) reduce then to the form:

$$
\begin{aligned}
& v^{\prime}+f v+W e v v^{\prime}-u^{2}+\frac{\sin \xi}{\xi} s-M u=\xi\left(u \frac{\partial u}{\partial \xi}-v \frac{\partial f}{\partial \xi}\right) \\
& \frac{t^{\prime}}{\operatorname{Pr}}+f t=\xi\left(u \frac{\partial s}{\partial \xi}-t \frac{\partial f}{\partial \xi}\right)
\end{aligned}
$$

where primes denote differentiation with respect to $\eta$. In terms of the dependent variables, the boundary conditions (16) become:

At $\eta=0, \quad f=0, \quad f^{\prime}=S_{f} f^{\prime \prime}(0), \quad \theta=1+S_{T} \theta^{\prime}(0)$

As $\eta \rightarrow \infty, \quad f^{\prime} \rightarrow 0, \quad \theta \rightarrow 0$

A two-dimensional computational mesh (grid) is imposed on the $\xi-\eta$ plane as shown in Fig.2. The stepping process is defined by:

$$
\begin{aligned}
& \eta_{0}=0, \eta_{j}=\eta_{j-1}+h_{j}, j=1,2, \ldots, J, \eta_{J} \equiv \eta_{\infty} \\
& \xi^{0}=0, \xi^{n}=\xi^{n-1}+k_{n}, n=1,2, \ldots, N .
\end{aligned}
$$

where $k_{n}$ and $h_{j}$ denote the step distances in the $\xi$ and $\eta$ directions respectively.

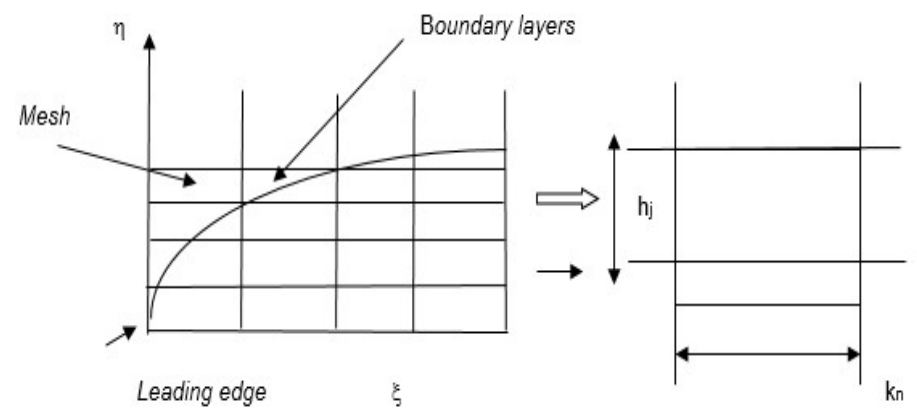

Fig. 2 Keller Box element and boundary layer mesh

If $g_{j}^{n}$ denotes the value of any variable at $\left(\eta_{j}, \xi^{n}\right)$, then the variables and derivatives of Equations. (19) - (23) at $\left(\eta_{j-1 / 2}, \xi^{n-1 / 2}\right)$ are replaced by:

$g_{j-1 / 2}^{n-1 / 2}=\frac{1}{4}\left(g_{j}^{n}+g_{j-1}^{n}+g_{j}^{n-1}+g_{j-1}^{n-1}\right)$,

$\left(\frac{\partial g}{\partial \eta}\right)_{j-1 / 2}^{n-1 / 2}=\frac{1}{2 h_{j}}\left(g_{j}^{n}-g_{j-1}^{n}+g_{j}^{n-1}-g_{j-1}^{n-1}\right)$,

$\left(\frac{\partial g}{\partial \xi}\right)_{j-1 / 2}^{n-1 / 2}=\frac{1}{2 k^{n}}\left(g_{j}^{n}-g_{j-1}^{n}+g_{j}^{n-1}-g_{j-1}^{n-1}\right)$,

The finite-difference approximation of equations. (19) - (23) for the mid-point $\left(\eta_{j-1 / 2}, \xi^{n}\right)$ assume the form given below:

$h_{j}^{-1}\left(f_{j}^{n}-f_{j-1}^{n}\right)=u_{j-1 / 2}^{n}$,

$h_{j}^{-1}\left(u_{j}^{n}-u_{j-1}^{n}\right)=v_{j-1 / 2}^{n}$, 


$$
\begin{aligned}
& h_{j}^{-1}\left(s_{j}^{n}-s_{j-1}^{n}\right)=t_{j-1 / 2}^{n}, \\
& \left(v_{j}-v_{j-1}\right)+(1+\alpha) \frac{h_{j}}{4}\left[\left(f_{j}+f_{j-1}\right)\left(v_{j}+v_{j-1}\right)\right] \\
& -\frac{(1+\alpha) h_{j}}{4}\left(u_{j}+u_{j-1}\right)^{2}+\frac{A h_{j}}{2}\left(s_{j}+s_{j-1}\right)+ \\
& \frac{W e}{2}\left(v_{j}+v_{j-1}\right)\left(v_{j}-v_{j-1}\right)-\frac{M h_{j}}{2}\left(u_{j}+u_{j-1}\right) \\
& -\frac{\alpha h_{j}}{2} f_{j-1 / 2}^{n-1}\left(v_{j}+v_{j-1}\right)+\frac{\alpha h_{j}}{2} v_{j-1 / 2}^{n-1}\left(f_{j}+f_{j-1}\right)=\left[R_{1}\right]_{j-1 / 2}^{n-1} \\
& \frac{1}{\operatorname{Pr}}\left(t_{j}-t_{j-1}\right)+(1+\alpha) \frac{h_{j}}{4}\left[\left(f_{j}+f_{j-1}\right)\left(t_{j}+t_{j-1}\right)\right] \\
& -\frac{\alpha h_{j}}{4}\left[\left(u_{j}+u_{j-1}\right)\left(s_{j}+s_{j-1}\right)\right]+\frac{\alpha h_{j}}{2} s_{j-1 / 2}^{n-1}\left(u_{j}+u_{j-1}\right) \\
& -\frac{\alpha h_{j}}{2} u_{j-1 / 2}^{n-1}\left(s_{j}+s_{j-1}\right)-\frac{\alpha h_{j}}{2} f_{j-1 / 2}^{n-1}\left(t_{j}+t_{j-1}\right) \\
& +\frac{\alpha h_{j}}{2} t_{j-1 / 2}^{n-1}\left(f_{j}+f_{j-1}\right)=\left[R_{2}\right]_{j-1 / 2}^{n-1}
\end{aligned}
$$

Here the following abbreviations apply:

$$
\begin{aligned}
& \alpha=\frac{\xi^{n-1 / 2}}{k_{n}}, A=\frac{\sin \left(\xi^{n-1 / 2}\right)}{\xi^{n-1 / 2}} \\
& {\left[R_{1}\right]_{j-1 / 2}^{n-1}=-h_{j}\left[\begin{array}{l}
\left.\left(\frac{v_{j}-v_{j-1}}{h_{j}}\right)+(1-\alpha)\left(f_{j-1 / 2} v_{j-1 / 2}\right)+W e v_{j-1} v_{j-1 / 2}^{\prime}\right] \\
+(1-\alpha)\left(u_{j-1 / 2}\right)^{2}+A\left(s_{j-1 / 2}\right)-M\left(u_{j-1 / 2}\right)
\end{array}\right]} \\
& {\left[R_{2}\right]_{j-1 / 2}^{n-1}=-h_{j}\left[\frac{1}{\operatorname{Pr}}\left(\frac{t_{j}-t_{j-1}}{h_{j}}\right)+(1-\alpha)\left(f_{j-1 / 2} t_{j-1 / 2}\right)+\alpha\left(u_{j-1 / 2} s_{j-1 / 2}\right)\right]}
\end{aligned}
$$

The boundary conditions take the form:

$$
f_{0}^{n}=u_{0}^{n}=0, \theta_{0}^{n}=1, u_{J}^{n}=0, v_{J}^{n}=0, \theta_{J}^{n}=0
$$

The emerging non-linear system of algebraic equations is linearized by means of Newton's method and then solved by the blockelimination method. The accuracy of computations is influenced by the number of mesh points in both directions. After experimenting with various grid sizes in the $\eta$-direction (radial coordinate) a larger number of mesh points are selected whereas in the $\xi$ direction (tangential coordinate) significantly less mesh points are utilized. $\eta_{\max }$ has been set at 10 and this defines a sufficiently large value at which the prescribed boundary conditions are satisfied. $\xi_{\max }$ is set at 3.0 for this flow domain. Mesh independence is therefore achieved in the present computations. The computer program of the algorithm is executed in MATLAB running on a PC.

If we assume $f_{j-1}^{n-1}, u_{j-1}^{n-1}, v_{j-1}^{n-1}, s_{j-1}^{n-1}, t_{j-1}^{n-1}$ to be known for $0 \leq j \leq J$, Eqs.(30) - (34) are a system of $5 J+5$ equations for the solution of $5 J+5$ unknowns $f_{j}^{n}, u_{j}^{n}, v_{j}^{n}, s_{j}^{n}, t_{j}^{n}, j=0,1,2 \ldots J$. This nonlinear system of algebraic equations is linearized by means of Newton's method which then solved in a very efficient manner by using the Keller-box method, which has been used most efficiently by Cebeci and Bradshaw (1984), taking the initial interaction with a given set of converged solutions at $\xi=\xi^{n}$. To initiate the process with $\xi=0$, we first prescribe a set of guess profiles for the functions $f, u, v, \theta$ and $t$ which are unconditionally convergent. These profiles are then employed in the Keller-box scheme with second-order accuracy to compute the correct solution step by step along the boundary layer. For a given $\xi$ the iterative procedure is stopped to give the final velocity and temperature distribution when the difference in computing these functions in the next procedure become less than $10^{-5}$, i.e., $\left|\delta f^{i}\right| \leq 10^{-5}$, where the superscript $i$ denotes the number of iterations.

For laminar flows the rate of convergence of the solutions of the equations (30) - (34) is quadratic provided the initial estimate to the desired solution is reasonably close to the final solution. Calculations are performed with four different $\Delta \eta$ spacing show that the rate of convergence of the solutions is quadratic in all cases for these initial profiles with typical iterations. The fact that Newton's method is used to linearize the non-linear algebraic equations and that with proper initial guess $\xi_{n}$ usually obtained from a solution at $\xi_{n-1}$, the rate of convergence of the solutions should be quadratic can be used to test the code for possible programming errors and to aid in the choice of $\Delta \xi$ spacing in the downstream direction. To study the effect of $\Delta \xi$ spacing on the rate of convergence of solutions, calculations were performed in the range $0 \leq \xi \leq 0.4$ with uniform $\Delta \xi$ spacing correspond ing to $0.08,0.04,0.02$ and 0.01 . Except for the results obtained with $\Delta \xi=0.08$, the rate of convergence of the solutions was essentially quadratic at each $\xi$ station. In most laminar boundary layer flows, a step size of $\Delta y=0.02$ to 0.04 is sufficient to provide accurate and comparable results. In fact in the present problem, we can even go up to $\Delta y=0.1$ and still get accurate and comparable results. This particular value of $\Delta y=0.1$ has also been used successfully by Merkin (1976). A uniform grid across the boundary is quite satisfactory for most laminar flow calculations, especially in laminar boundary layer. However, the Keller-box method is unique in which various spacing in both $\eta$ and $\xi$ directions can be used (Aldoss et al.,(1996).

\section{VALIDATION OF KELLER BOX SOLUTIONS}

The present Keller box solutions have been validated for the special case of non-magnetic $(M=0)$ Newtonian flow $(W e=0)$ in the absence of thermal and partial slip $\left(S_{f}=S_{T}=0\right)$. It is also possible to make a comparison as the momentum equation and boundary conditions assume the following reduced form:

$$
\begin{aligned}
& f^{\prime \prime \prime}+f f^{\prime \prime}-f^{\prime 2}+\frac{\sin \xi}{\xi} \theta=\xi\left(f^{\prime} \frac{\partial f^{\prime}}{\partial \xi}-f^{\prime \prime} \frac{\partial f}{\partial \xi}\right) \\
& \text { At } \quad \eta=0 ; f=0 ; f^{\prime}=0, \theta=1 \\
& \text { At } \quad \eta \rightarrow \infty: f^{\prime} \rightarrow 0 ; \theta \rightarrow 0
\end{aligned}
$$

The energy equation (15) is identical to that considered in Merkin (1976), Molla et al. (2011) and Javed et al. (2015). The comparison of solutions is documented in Table 1.1 \& 1.2 Excellent correlation is achieved and confidence in the present solutions is therefore justifiably high.

Table 1.1: Variations of the local heat transfer coefficient $C_{f}$ for various values of $\xi$ with $W e=S_{f}=S_{T}=M=0.0$

\begin{tabular}{|c|c|c|c|c|}
\hline \multirow{2}{*}{$\xi$} & \multicolumn{4}{|c|}{$C_{f}$} \\
\cline { 2 - 5 } & $\begin{array}{c}\text { Merkin } \\
(1976)\end{array}$ & $\begin{array}{c}\text { Molla et al. } \\
(2011)\end{array}$ & $\begin{array}{c}\text { Javed et al. } \\
(2015)\end{array}$ & $\begin{array}{c}\text { Present } \\
\text { Solutions }\end{array}$ \\
\hline 0 & 0.0000 & 0.0000 & 0.0000 & 0.0000 \\
\hline$\pi / 6$ & 0.4151 & 0.4139 & 0.4150 & 0.4247 \\
\hline$\pi / 3$ & 0.7558 & 0.7527 & 0.7557 & 0.7559 \\
\hline$\pi / 2$ & 0.9579 & 0.9526 & 0.9578 & 0.9576 \\
\hline $2 \pi / 3$ & 0.9756 & 0.9677 & 0.9555 & 0.9551 \\
\hline $5 \pi / 6$ & 0.7822 & 0.7717 & 0.7822 & 0.7821 \\
\hline$\pi$ & 0.3391 & 0.3238 & 0.3388 & 0.3385 \\
\hline
\end{tabular}


Table 1.2: Variations of the local heat transfer coefficient $N u$ for various values of $\xi$ with $W e=S_{f}=S_{T}=M=0.0$

\begin{tabular}{|c|c|c|c|c|}
\hline \multirow{2}{*}{$\xi$} & \multicolumn{4}{|c|}{$N u$} \\
\cline { 2 - 5 } & $\begin{array}{c}\text { Merkin } \\
(1976)\end{array}$ & $\begin{array}{c}\text { Molla et al. } \\
(2011)\end{array}$ & $\begin{array}{c}\text { Javed et al. } \\
(2015)\end{array}$ & $\begin{array}{c}\text { Present } \\
\text { Solutions }\end{array}$ \\
\hline 0 & 0.4214 & 0.4216 & 0.4215 & 0.4218 \\
\hline$\pi / 6$ & 0.4161 & 0.4163 & 0.4163 & 0.4265 \\
\hline$\pi / 3$ & 0.4007 & 0.4006 & 0.4009 & 0.4011 \\
\hline$\pi / 2$ & 0.3745 & 0.3741 & 0.3747 & 0.3752 \\
\hline $2 \pi / 3$ & 0.3364 & 0.3355 & 0.3355 & 0.3354 \\
\hline $5 \pi / 6$ & 0.2825 & 0.2810 & 0.2824 & 0.2839 \\
\hline$\pi$ & 0.1945 & 0.1911 & 0.1943 & 0.1954 \\
\hline
\end{tabular}

\section{RESULTS AND DISCUSSION}

Extensive computations have been conducted using the Keller box code to study the influence of the key thermo-physical parameters on velocity, temperature, skin friction and Nusselt number. These are visualized in figs. 3a-b to $\mathbf{1 2} \mathbf{a}-\mathbf{b}$. In the present computations, the following default parameters are prescribed (unless otherwise stated): $W e=0.3, \operatorname{Pr}=7.0, \quad M=1.0, S_{f}=0.5, S_{T}=1.0, \xi=1.0$.

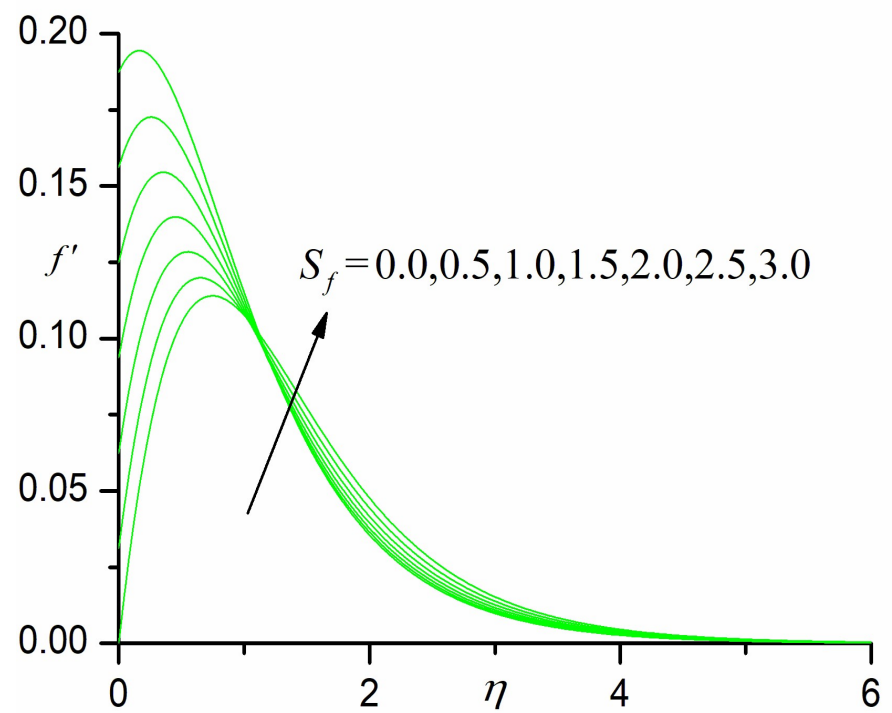

Fig. 3a Effect of $S_{f}$ on velocity profiles

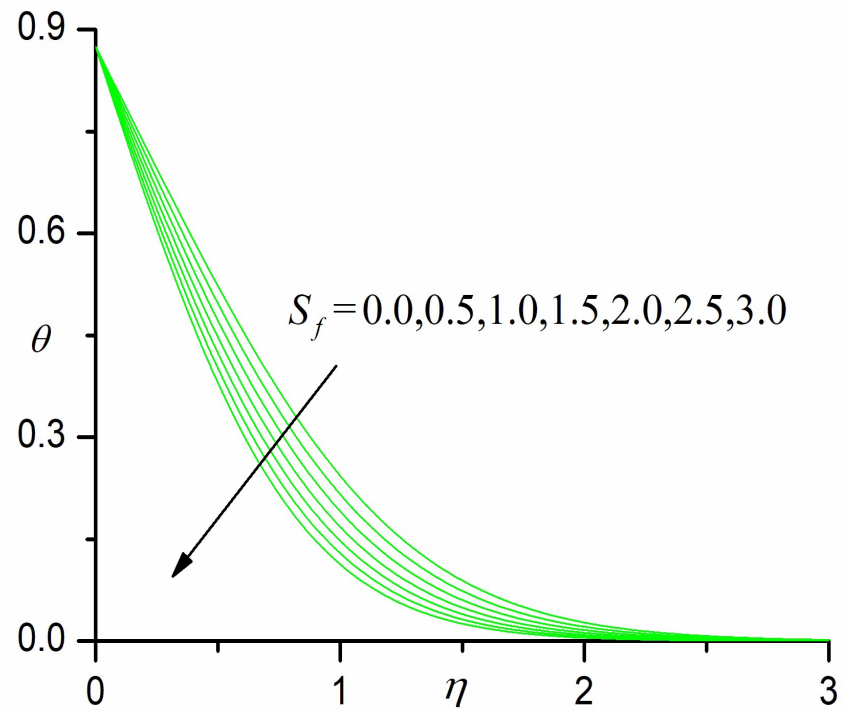

Fig. 3b Effect of $S_{f}$ on temperature profiles

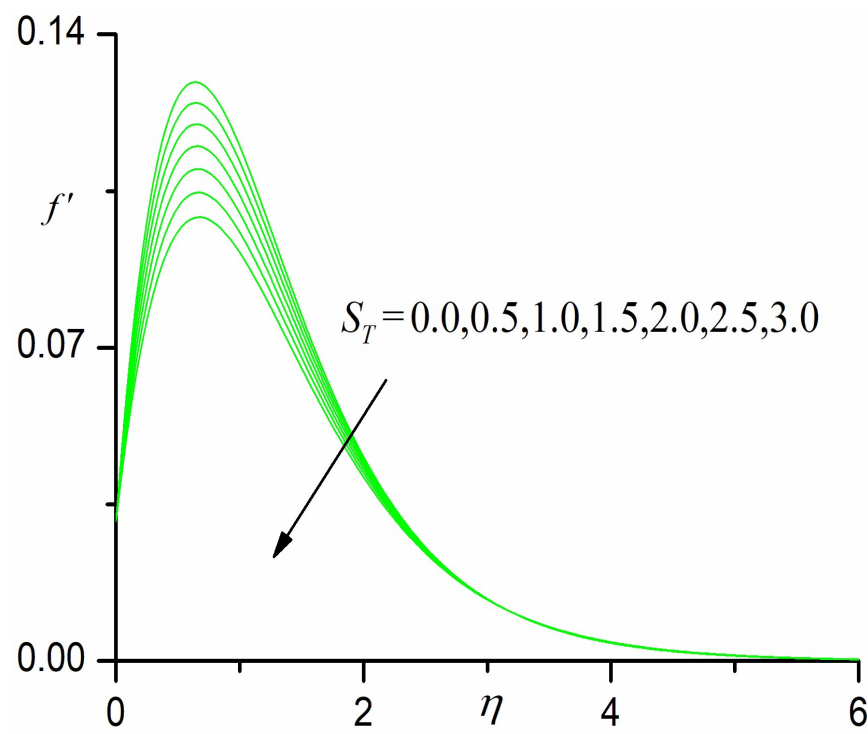

Fig. 4a Effect of $S_{T}$ on velocity profiles

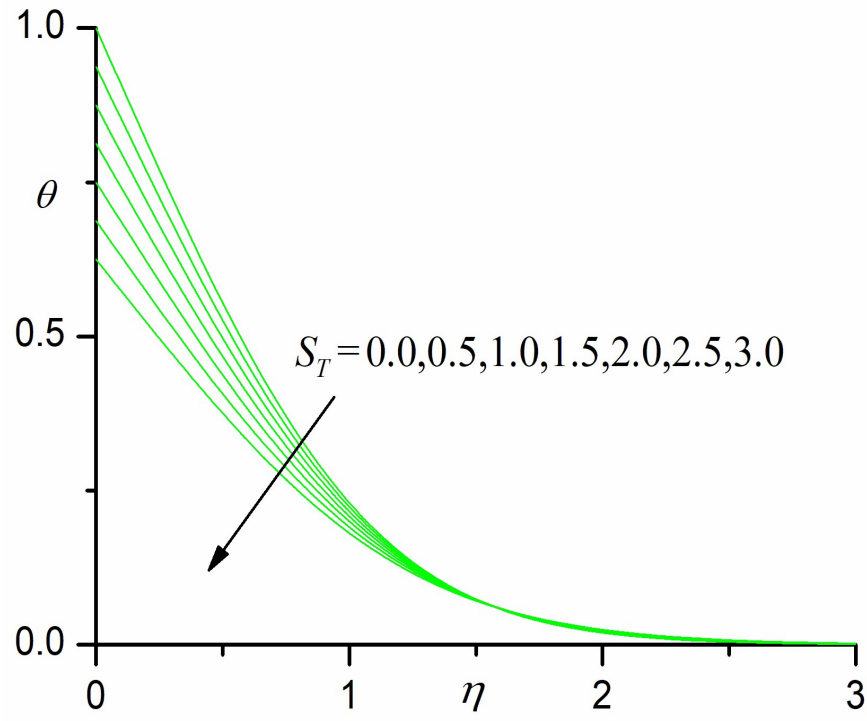

Fig. 4b Effect of $S_{T}$ on temperature profiles

Fig.3a-b presents the evolution in temperature function, $\theta(\eta)$, with transverse coordinate $\eta$ with variation in hydrodynamic slip parameter, $S_{f}$. Temperature profiles consistently decay monotonically from a maximum at the cylinder surface to the free stream. All profiles converge at a large value of transverse coordinate, again showing that a sufficiently large infinity boundary condition has been utilized in the numerical computations. Greater momentum slip substantially increases temperatures in the boundary layer and therefore also elevates thermal boundary layer thickness. The regime is therefore coolest when slip is absent $\left(S_{f}=0\right.$ i.e. no-slip classical case $)$ and hottest with strong hydrodynamic wall slip.

Figs. $4 a$ and $4 \mathbf{b}$ illustrate the influence of thermal slip parameter $\left(S_{T}\right)$ on the velocity and temperature. Both velocity and temperature are consistently suppressed with an increase in $S_{T}$. Temperatures are strongly depressed in particular at the cylinder surface. Greater thermal jump therefore decelerates the flow and cools the boundary layer. Momentum boundary layer thickness is enhanced whereas thermal boundary layer thickness is decreased with increasing thermal slip. A similar response has been observed by Basir et al. (2016). Physically, as the thermal slip parameter rises, the fluid flow within the boundary layer becomes progressively less sensitive to the 
heating effects at the cylinder surface and a decreased quantity of thermal energy (heat) is transferred from the hot cylinder surface to the fluid, resulting in a fall in temperatures, manifesting in a cooling and thinning of the thermal boundary layer. This has important implications in thermal polymer enrobing, since thermal slip modifies the heat transferred to the polymer material which in turn alters characteristics of the final product (1990).

Fig.5a-b illustrates that the velocity is reduced with an increase in $M$. This indicates that the transverse magnetic field opposes the transport phenomena since an increase in $M$ leads to an increase in the Lorentz force, which opposes the transport process. This stronger Lorentz force produces more resistance to the transport. The higher the value of $M$, the more prominent is the reduction in hydrodynamic boundary layer thickness. But from Figs.5b, the opposite phenomenon is observed with an increase in Magnetic field parameter $M$ on temperature filed. The excess work expended in dragging the polymer against the action of the magnetic field is dissipated as thermal energy (heat). This energizes the boundary layer and increases thermal boundary layer thickness. Again the influence of magnetic field is sustained throughout the entire boundary layer domain. These results concur with other investigations of magnetic non-Newtonian heat transfer including Kasim et al. (2013) and Megahed (2012).

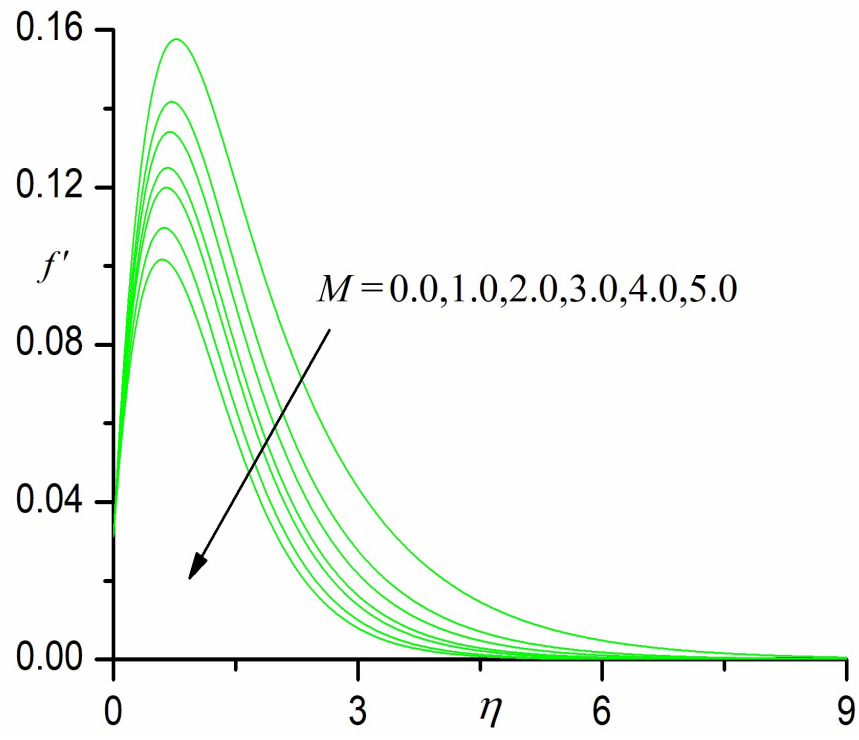

Fig. 5a Effect of $M$ on velocity profiles

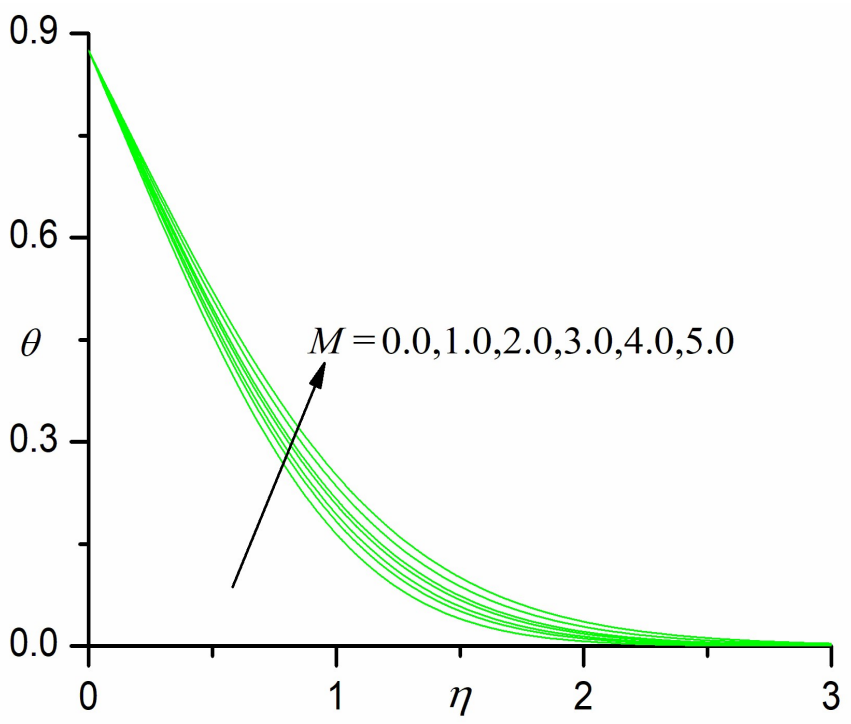

Fig. 5b Effect of $M$ on temperature profiles

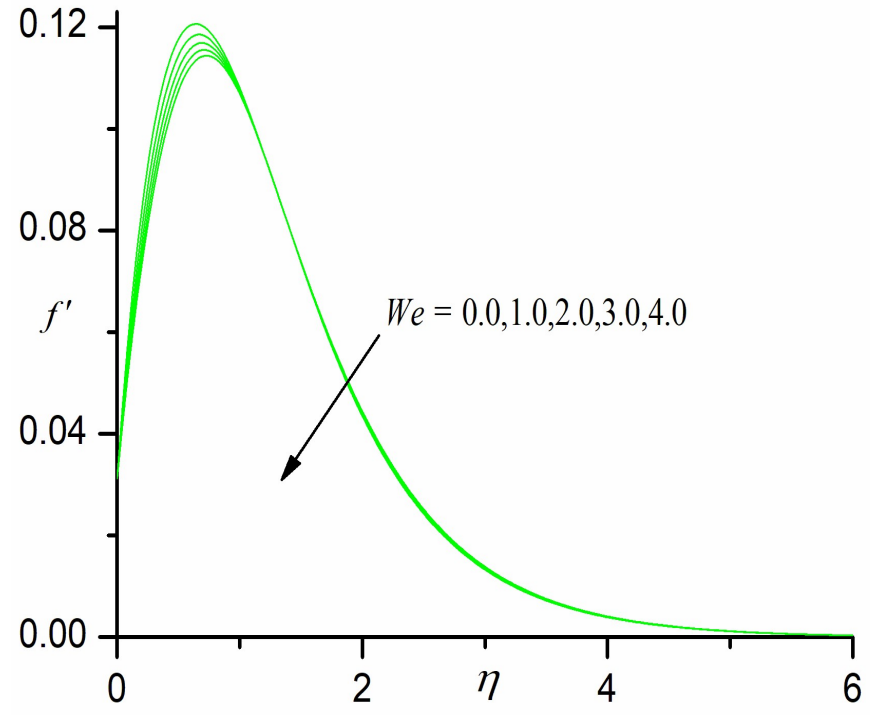

Fig. 6a Effect of $W e$ on velocity profiles

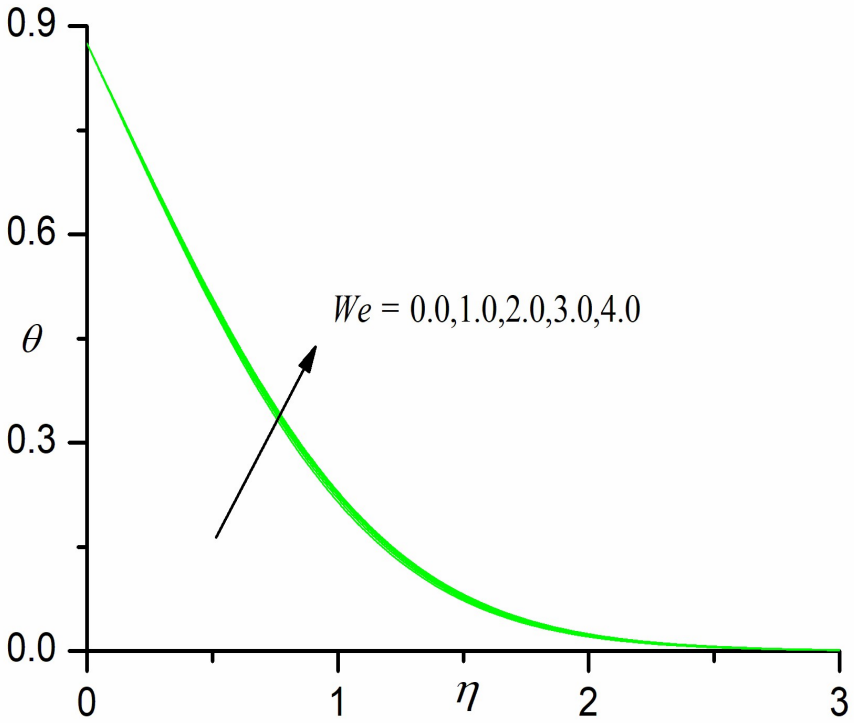

Fig. 6b Effect of We on temperature profiles

Figs. 6a-b, depict the effect of Williamson viscoelastic fluid parameter, $W e$ on velocity and temperature profiles. It is shown that the effect of $W e$ reduces velocity near the cylinder surface but depletes it further away. Increasing Williamson viscoelastic fluid parameter however consistently weakly increases temperatures throughout the boundary layer. The influence on velocity field is significantly greater however since the viscoelastic effect is simulated solely in the momentum equation (14) via the shear term (mixed derivative) $W e f^{\prime \prime} f^{\prime \prime \prime}$. Williamson viscoelastic fluid parameter $(\mathrm{We})$ measures the relative effects of viscosity to elasticity. Williamson viscoelastic fluid parameter of zero corresponds to a purely Newtonian fluid, and infinite Weissenberg number corresponds to a purely elastic solid. The effect of viscoelastic parameter is indirectly transmitted to the temperature field. Since the Williamson viscoelastic fluid parameter is also present in the wall boundary condition, the acceleration effect is only confined to the region close to the cylinder surface. Overall however the dominant influence of $W e$, is near the wall and is found to be assistive to momentum development. Only a very small decrease in temperature is observed with a large enhancement in Weissenberg parameter, as shown in Fig.6b. Thermal boundary layer thickness is therefore enhanced with increasing $W e$ values i.e. decreasing viscosity and 
increasing elastic effects. Effectively therefore Newtonian fluids ( We $=0$ ) achieve lower velocities and temperatures than Williamson fluids. Similar trends have been reported by Hayat et al. (2016) and Khan and Khan (2014).

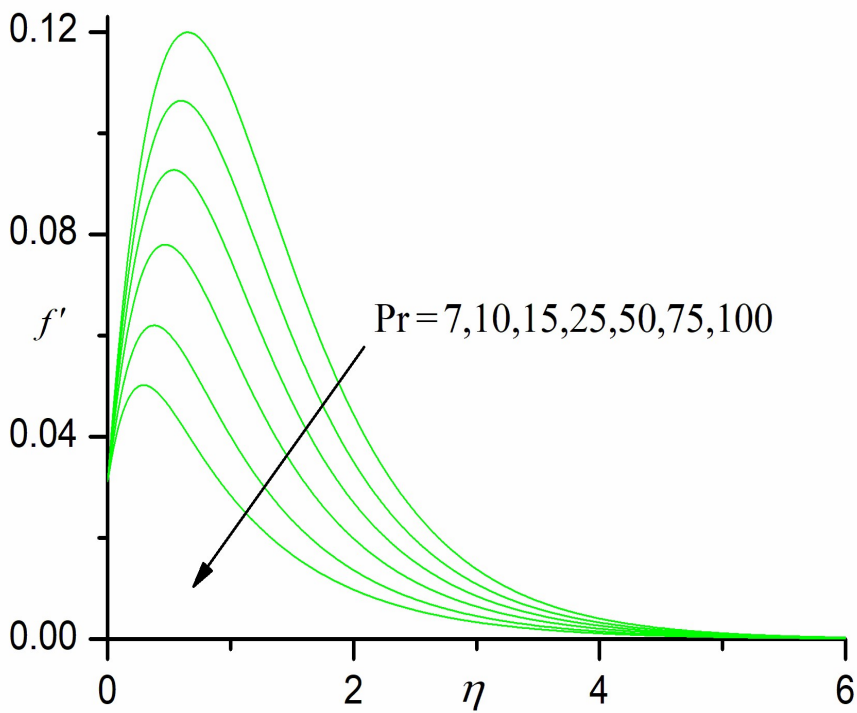

Fig. 7a Effect of $\operatorname{Pr}$ on velocity profiles

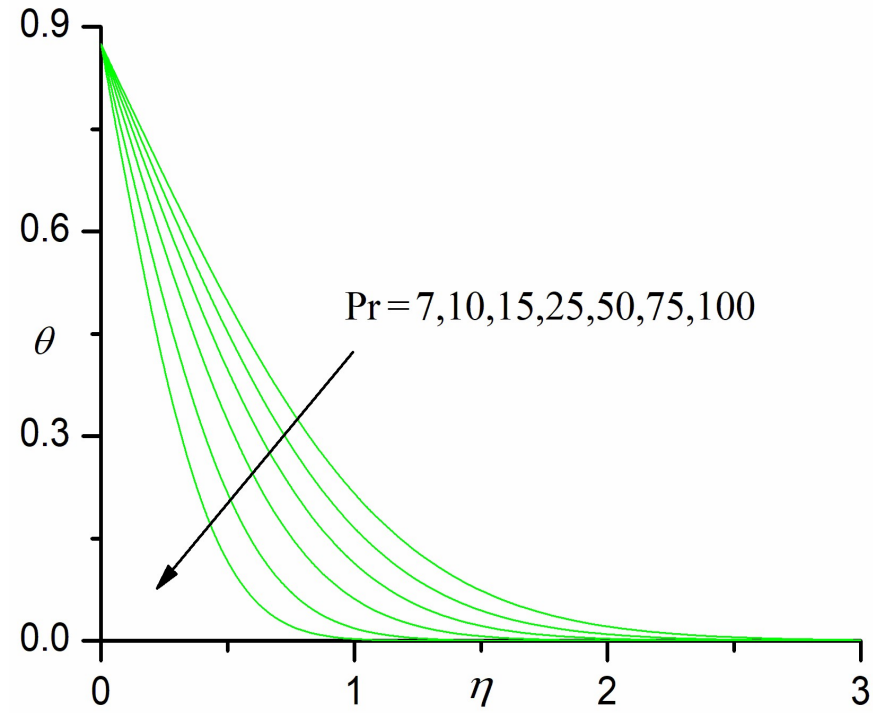

Fig. 7b Effect of $\operatorname{Pr}$ on temperature profiles

Figs. 7a $-7 \mathbf{7 b}$, present the impact of Prandtl number $(\mathrm{Pr})$ on the velocity and temperature profiles along the transverse coordinate i.e. normal to the cylinder surface. Prandtl number epitomizes the ratio of momentum diffusion to thermal diffusion in the boundary layer regime. It also represents the ratio of the product of specific heat capacity and dynamic viscosity, to the fluid thermal conductivity. For $\mathrm{Pr}$ equal to unity both the momentum and thermal diffusion rates are the same, as are the momentum and thermal boundary layer thicknesses. An increment in $\operatorname{Pr}$ from 7.0 through 10, 15, 25, 50, 75 to 100, which corresponds to increasing momentum diffusivity and decreasing thermal diffusivity, results in a tangible reduction in velocity magnitudes throughout the boundary layer. For $\operatorname{Pr}<1$, thermal diffusivity surpasses momentum diffusivity i.e. heat will diffuse at a faster rate than momentum. In this manner for lower $\mathrm{Pr}$ fluids (e.g. $\mathrm{Pr}$ $=0.01$ which physically relate to liquid metals), the flow will be accelerates whereas for greater $\operatorname{Pr}$ fluids (e.g. $P r=1$ for low weight molecular polymers $(2004,2015)$ it will be strongly decelerated, as observed in Fig.7b. For $\mathrm{Pr}<1$, the momentum boundary layer thickness is lesser than thermal boundary layer thickness. The asymptotically smooth profiles in the free stream (high $\eta$ values) confirm that an adequately large infinity boundary condition has been imposed in the Keller box numerical code.

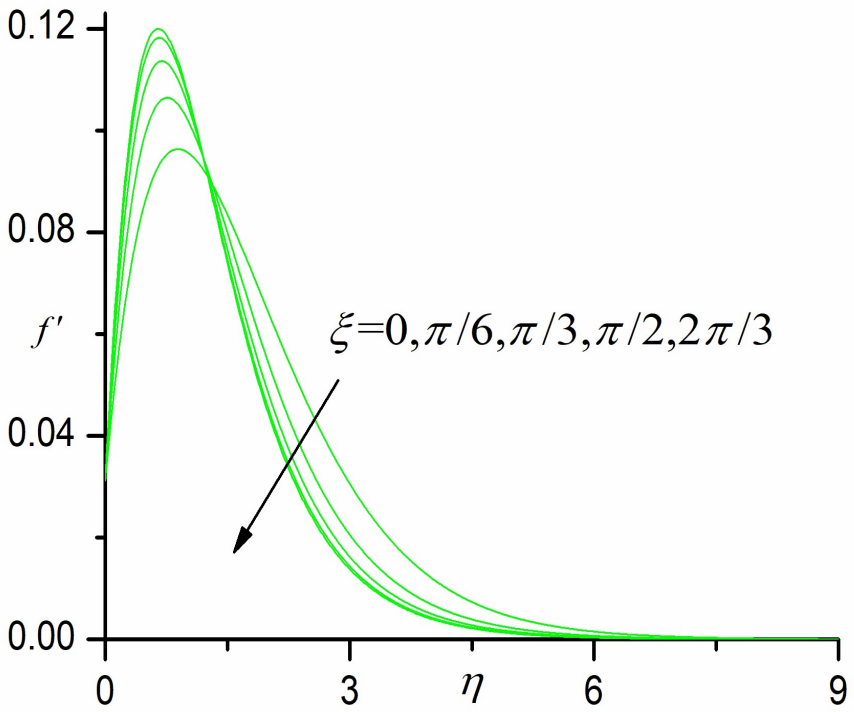

Fig. 8a Effect of $\xi$ on velocity profiles

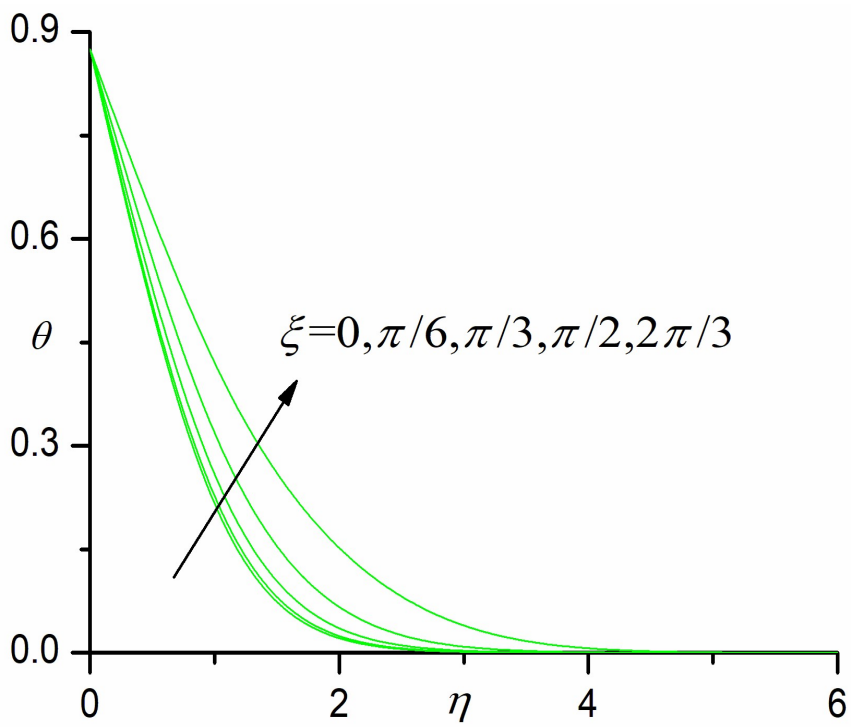

Fig. 8b Effect of $\xi$ on temperature profiles

Figs. 8a-b illustrate the influence of the stream wise (tangential) coordinate, $\xi$, on the velocity and temperature distributions. A weak deceleration in the boundary layer flow is experienced with greater $\xi$, values i.e. with progressive distance along the cylinder surface from the lower stagnation point $(\xi=0)$, as shown in fig. $8 \mathrm{a}$ Momentum boundary layer thickness is therefore increased marginally with $\xi$ values. Conversely a weak enhancement in temperature is computed in fig. $8 \mathrm{~b}$, with increasing $\xi$ values. Thermal boundary layer thickness is increased therefore as we progress from the lower stagnation point on the cylinder surface around the cylinder periphery upwards.

Figs. 9a-b presents the variation in surface shear stress (skin friction) and Nusselt number (wall heat transfer gradient) with Weissenberg number with both thermal and velocity slip present. In consistency with the near-wall behaviour computed for the velocity field in fig. 6a, there is a significant elevation in skin friction with increasing $W e$ values. With progressively greater $W e$ values the elasticity in the polymer is increased. This aids in momentum 
development and accelerates the boundary layer flow. A similar trend has been computed in the studies by Hayat et al. (2016). The Weissenberg number indicates the degree of anisotropy or orientation generated by the deformation, and is appropriate to describe flows with a constant stretch history, and therefore appropriate for polymers. A strong reduction in Nusselt number arises with an elevation in Weissenberg number i.e. heat is transferred from the cylinder surface to the boundary layer. This concurs with fig. $6 \mathrm{~b}$ wherein temperature (and thermal boundary layer thickness) are found to be enhanced with Weissenberg number. The cylinder surface is therefore effectively cooled with greater Weissenberg numbers.

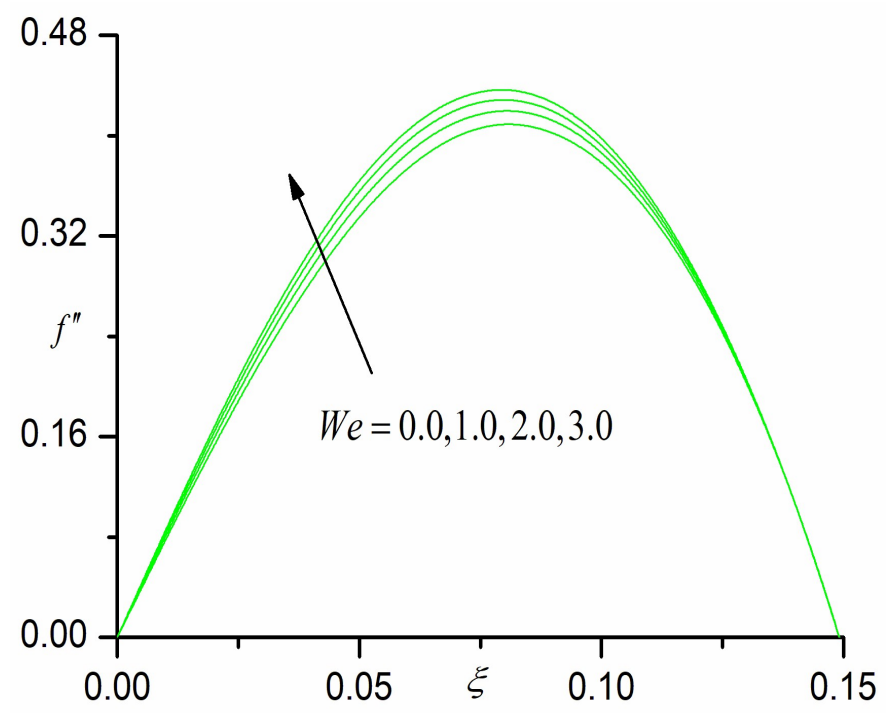

Fig. 9a Skin friction profiles for various values of $W e$

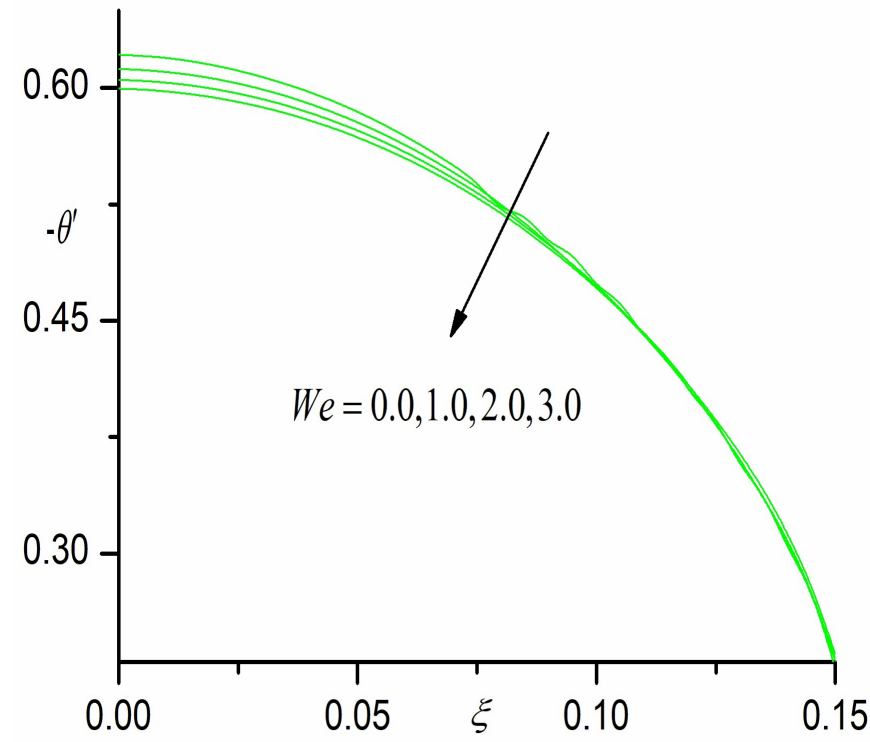

Fig. 9b Nusselt number profiles for various values of $W e$

Figs. 10a-b illustrate the skin friction and Nusselt number distributions with various values of momentum slip parameter $\left(S_{f}\right)$. A marked depreciation in skin friction is observed with greater momentum slip (fig. 10a). Conversely a strong elevation in Nusselt number is generated with greater momentum slip effect (fig. 10b). In both plots the profiles never intersect i.e. the momentum slip effect is consistent for all values of stream wise parameter $(\xi)$. The influence of momentum (hydrodynamic) slip is non-trivial and demonstrates that a sizeable modification in surface thermo fluid characteristics is induced with slip and indeed that the methodology employed to simulate it quite realistically simulates real macroscopic effects of certain molecular phenomena at polymer/solid interfaces.

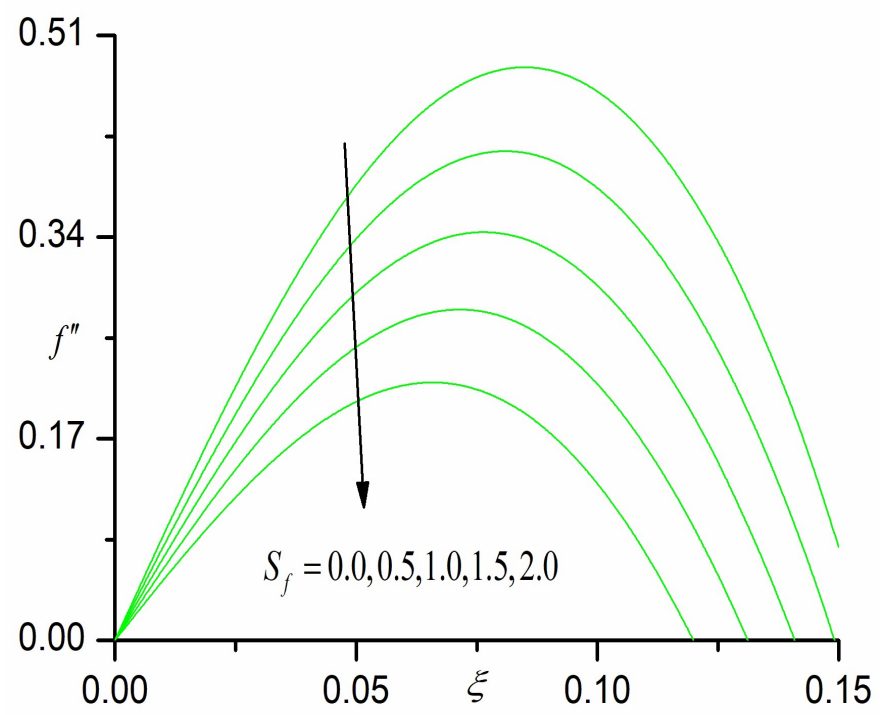

Fig. 10a Skin friction profiles for various values of $S_{f}$

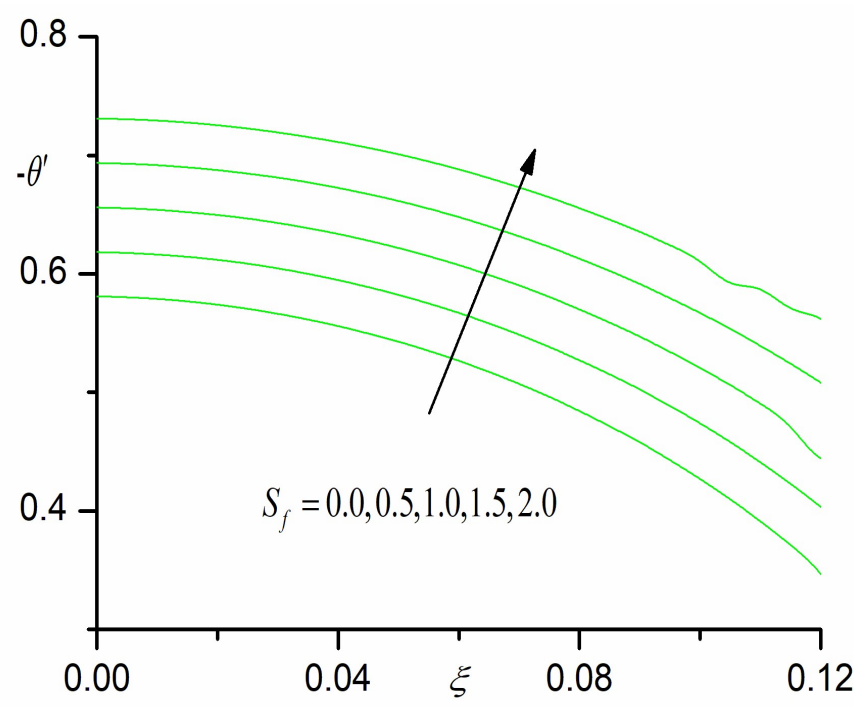

Fig. 10b Nusselt number profiles for various values of $S_{f}$

Table 2: Values of $f^{\prime \prime}(\xi, 0)$ for different $S_{f}, S_{T}$ and $\xi$

\begin{tabular}{|c|c|c|c|c|}
\hline \multirow{2}{*}{$S_{f}$} & \multirow{2}{*}{$S_{T}$} & \multicolumn{3}{|c|}{$f^{\prime \prime}(\xi, 0)$} \\
\hline & & $\xi=0.0$ & $\xi=\pi / 6$ & $\xi=\pi / 4$ \\
\hline 0.0 & \multirow{7}{*}{1.0} & 0 & 0.1908 & 0.2592 \\
\hline 0.1 & & 0 & 0.1858 & 0.2522 \\
\hline 0.2 & & 0 & 0.1807 & 0.2453 \\
\hline 0.3 & & 0 & 0.1757 & 0.2383 \\
\hline 0.5 & & 0 & 0.1657 & 0.2244 \\
\hline 0.8 & & 0 & 0.1506 & 0.2036 \\
\hline 1.0 & & 0 & 0.1406 & 0.1897 \\
\hline \multirow{6}{*}{0.5} & 0.0 & 0 & 0.1871 & 0.2536 \\
\hline & 0.5 & 0 & 0.1765 & 0.2391 \\
\hline & 1.5 & 0 & 0.1547 & 0.2096 \\
\hline & 2.0 & 0 & 0.1437 & 0.1945 \\
\hline & 2.5 & 0 & 0.1324 & 0.1792 \\
\hline & 3.0 & 0 & 0.1210 & 0.1636 \\
\hline
\end{tabular}


Table 3: Values of $-\theta^{\prime}(\xi, 0)$ for different $S_{f}, S_{T}$ and $\xi$

\begin{tabular}{|c|c|c|c|c|}
\hline \multirow{2}{*}{$S_{f}$} & \multirow{2}{*}{$S_{T}$} & \multicolumn{3}{|c|}{$-\theta^{\prime}(\xi, 0)$} \\
\hline & & $\xi=0.0$ & $\xi=\pi / 6$ & $\xi=\pi / 4$ \\
\hline 0.0 & \multirow{7}{*}{1.0} & 0.7199 & 0.7015 & 0.6857 \\
\hline 0.1 & & 0.7326 & 0.7140 & 0.6981 \\
\hline 0.2 & & 0.7454 & 0.7266 & 0.7105 \\
\hline 0.3 & & 0.7582 & 0.7393 & 0.7229 \\
\hline 0.5 & & 0.7839 & 0.7645 & 0.7475 \\
\hline 0.8 & & 0.8225 & 0.8026 & 0.7850 \\
\hline 1.0 & & 0.8483 & 0.8279 & 0.8100 \\
\hline \multirow{6}{*}{0.5} & 0.0 & 0.9259 & 0.9032 & 0.8835 \\
\hline & 0.5 & 0.8543 & 0.8333 & 0.8151 \\
\hline & 1.5 & 0.7147 & 0.6979 & 0.6814 \\
\hline & 2.0 & 0.6469 & 0.6308 & 0.6166 \\
\hline & 2.5 & 0.5804 & 0.5659 & 0.5532 \\
\hline & 3.0 & 0.5154 & 0.5025 & 0.4911 \\
\hline
\end{tabular}

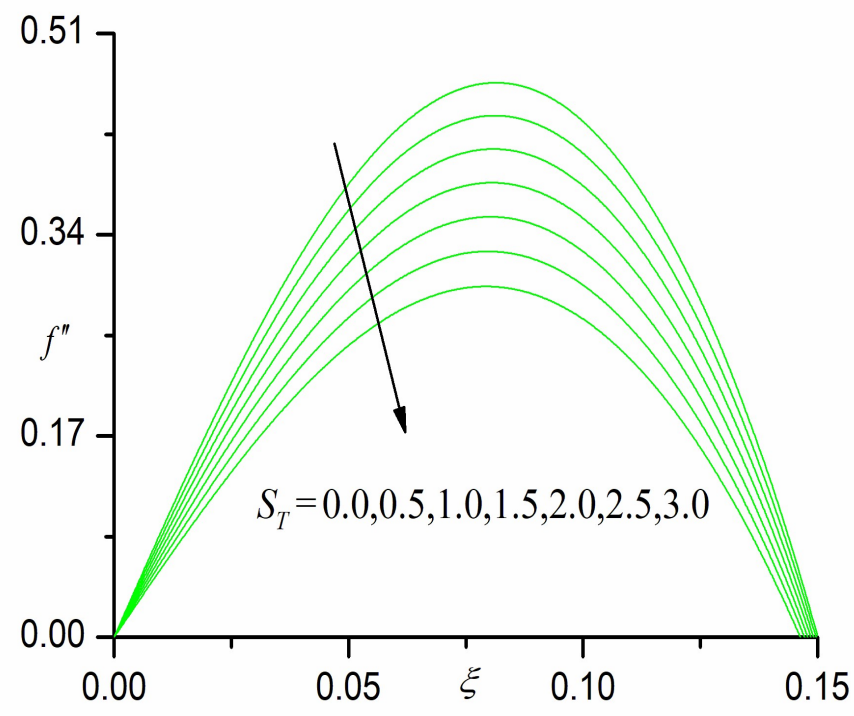

Fig. 11a Skin friction profiles for various values of $S_{T}$

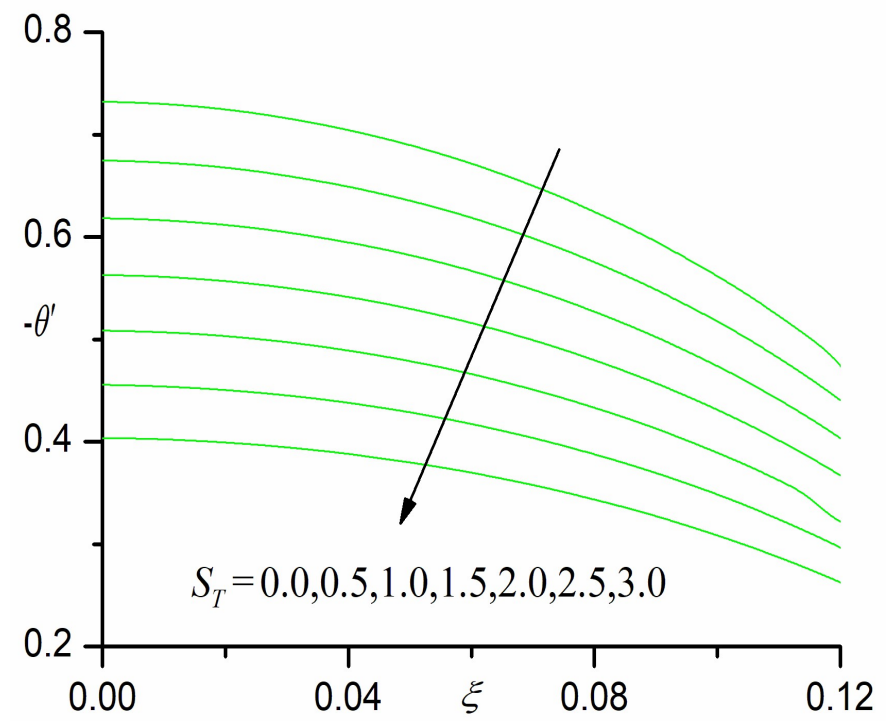

Fig. 11b Nusselt number profiles for various values of $S_{T}$

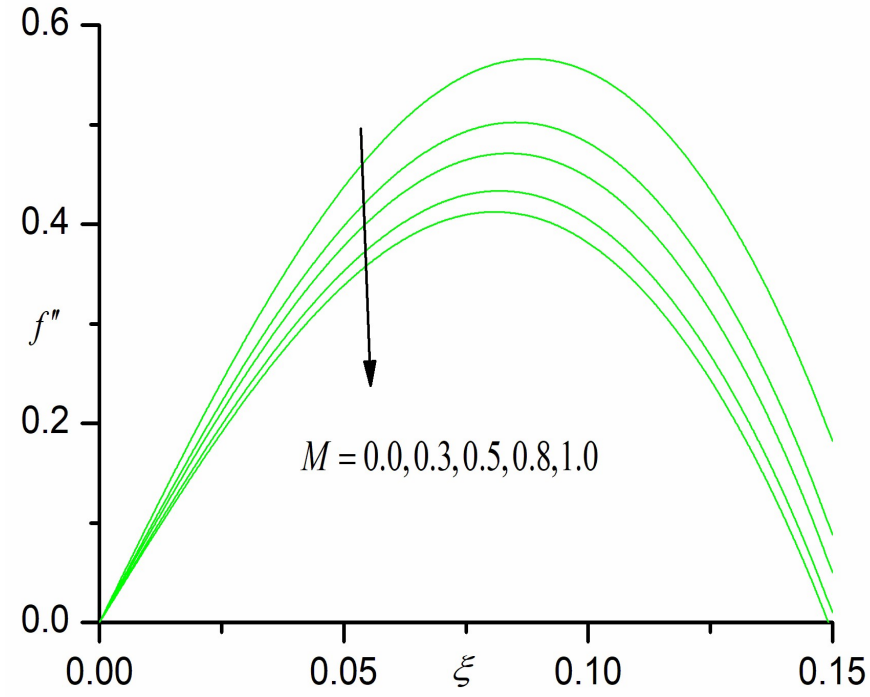

Fig. 12a Skin friction profiles for various values of $M$

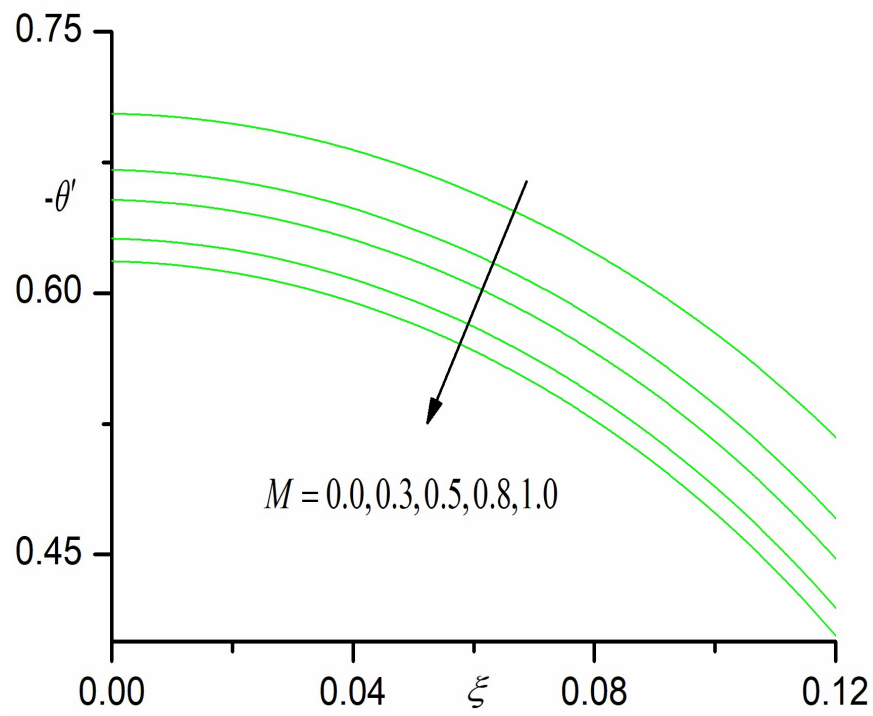

Fig. 12b Nusselt number profiles for various values of $M$

Figs. 11a-b present the distributions in skin friction and Nusselt number with thermal slip effect $\left(S_{T}\right)$. Both skin friction and Nusselt number are strongly reduced with an increase thermal slip. The boundary layer is therefore decelerated and heated with stronger thermal slip. With thermal slip absent therefore the skin friction is maximized at the cylinder surface. The inclusion of thermal slip, which is encountered in various slippy polymer flows, is therefore important in more physically realistic simulations.

Figs. 12a-b illustrate the influence of magnetic parameter $(M)$ on skin friction and Nusselt number. A significant depletion is caused in skin friction (fig. 12a) with greater magnetic field, which corresponds to a retardation of the boundary layer flow. The maximum skin friction therefore is achieved only in the absence of a radial magnetic field i.e. $M=0$. For $M<1$, the magnetic body force is exceeded by the viscous hydrodynamic force in the regime. For $M>1$ the contrary is the case. The reduction in Nusselt number with greater $M$ values implies that the transfer of heat from the boundary layer to the wall (cylinder surface) is reduced. This physically indicates therefore that greater heat is conveyed away from the cylinder surface to the fluid which explains the higher temperatures associated with strong magnetic field in the earlier computations (fig. 5b). Magnetic field is therefore a 
potent mechanism for controlling thermal and velocity characteristics in electrically-conducting polymer dynamics.

Table 4: Values of $f^{\prime \prime}(\xi, 0)$ for different $S_{f}, S_{T}$ and $\xi$

\begin{tabular}{|c|c|c|c|c|}
\hline \multirow{2}{*}{$S_{f}$} & \multirow{2}{*}{$S_{T}$} & \multicolumn{3}{|c|}{$f^{\prime \prime}(\xi, 0)$} \\
\hline & & $\xi=\pi / 3$ & $\xi=\pi / 2$ & $\xi=2 \pi / 3$ \\
\hline 0.0 & \multirow{7}{*}{1.0} & 0.3473 & 0.4415 & 0.4506 \\
\hline 0.1 & & 0.3376 & 0.4280 & 0.4344 \\
\hline 0.2 & & 0.3280 & 0.4144 & 0.4182 \\
\hline 0.3 & & 0.3183 & 0.4009 & 0.4020 \\
\hline 0.5 & & 0.2989 & 0.3738 & 0.3694 \\
\hline 0.8 & & 0.2699 & 0.3330 & 0.3204 \\
\hline 1.0 & & 0.2504 & 0.3058 & 0.2876 \\
\hline \multirow{6}{*}{0.5} & 0.0 & 0.3382 & 0.4241 & 0.4218 \\
\hline & 0.5 & 0.3187 & 0.3991 & 0.3957 \\
\hline & 1.5 & 0.2790 & 0.3481 & 0.3427 \\
\hline & 2.0 & 0.2587 & 0.3222 & 0.3157 \\
\hline & 2.5 & 0.2381 & 0.2958 & 0.2884 \\
\hline & 3.0 & 0.2172 & 0.2690 & 0.2606 \\
\hline
\end{tabular}

Table 5: Values of $-\theta^{\prime}(\xi, 0)$ for different $S_{f}, S_{T}$ and $\xi$

\begin{tabular}{|c|c|c|c|c|}
\hline \multirow{2}{*}{$S_{f}$} & \multirow{2}{*}{$S_{T}$} & \multicolumn{3}{|c|}{$-\theta^{\prime}(\xi, 0)$} \\
\hline & & $\xi=\pi / 3$ & $\xi=\pi / 2$ & $\xi=2 \pi / 3$ \\
\hline 0.0 & \multirow{7}{*}{1.0} & 0.6521 & 0.5687 & 0.4466 \\
\hline 0.1 & & 0.6641 & 0.5795 & 0.4554 \\
\hline 0.2 & & 0.6761 & 0.5904 & 0.4638 \\
\hline 0.3 & & 0.6883 & 0.6013 & 0.4727 \\
\hline 0.5 & & 0.7125 & 0.6234 & 0.4904 \\
\hline 0.8 & & 0.7492 & 0.6563 & 0.5173 \\
\hline 1.0 & & 0.7726 & 0.6786 & 0.5351 \\
\hline \multirow{6}{*}{0.5} & 0.0 & 0.8421 & 0.7374 & 0.5814 \\
\hline & 0.5 & 0.7767 & 0.6798 & 0.5355 \\
\hline & 1.5 & 0.6494 & 0.5678 & 0.4462 \\
\hline & 2.0 & 0.5875 & 0.5134 & 0.4029 \\
\hline & 2.5 & 0.5272 & 0.4601 & 0.3607 \\
\hline & 3.0 & 0.4680 & 0.4080 & 0.3194 \\
\hline
\end{tabular}

Table 6: Values of $f^{\prime \prime}(\xi, 0)$ for different values of $W e, M$ and $P r$

\begin{tabular}{|c|c|c|c|c|c|}
\hline \multirow{2}{*}{$W e$} & \multirow{2}{*}{$M$} & \multicolumn{4}{|c|}{$f^{\prime \prime}(\xi, 0)$} \\
\hline & & $\operatorname{Pr}=7$ & $\operatorname{Pr}=10$ & $\operatorname{Pr}=20$ & $\operatorname{Pr}=25$ \\
\hline 0.0 & \multirow{6}{*}{1.0} & 0.2960 & 0.2730 & 0.2294 & 0.2157 \\
\hline 0.5 & & 0.3008 & 0.2769 & 0.2316 & 0.2176 \\
\hline 1.0 & & 0.3049 & 0.2803 & 0.2341 & 0.2198 \\
\hline 2.0 & & 0.3122 & 0.2864 & 0.2382 & 0.2234 \\
\hline 3.0 & & 0.3185 & 0.2916 & 0.2417 & 0.2265 \\
\hline 4.0 & & 0.3240 & 0.2964 & 0.2449 & 0.2294 \\
\hline \multirow{6}{*}{0.3} & 0.0 & 0.3760 & 0.3444 & 0.2870 & 0.2698 \\
\hline & 0.5 & 0.3302 & 0.3029 & 0.2529 & 0.2376 \\
\hline & 1.0 & 0.2989 & 0.2753 & 0.2308 & 0.2170 \\
\hline & 2.0 & 0.2538 & 0.2352 & 0.1988 & 0.1872 \\
\hline & 3.0 & 0.2203 & 0.2052 & 0.1745 & 0.1645 \\
\hline & 4.0 & 0.1936 & 0.1809 & 0.1546 & 0.1457 \\
\hline
\end{tabular}

Tables 2-7 present numerical values for the influence of the various parameters on skin friction and Nusselt number functions.
These confirm the trends already elaborated in figs $9 \mathrm{a}-\mathrm{b}$ to $12 \mathrm{a}-\mathrm{b}$ and furthermore provide benchmarks against which other researchers may validate extensions of the present model.

Table 7: Values of $-\theta^{\prime}(\xi, 0)$ for different values of $W e, M$ and $P r$

\begin{tabular}{|c|c|c|c|c|c|}
\hline \multirow{2}{*}{$W e$} & \multirow{2}{*}{$M$} & \multicolumn{4}{|c|}{$-\theta^{\prime}(\xi, 0)$} \\
\hline & & $\operatorname{Pr}=7$ & $\operatorname{Pr}=10$ & $\operatorname{Pr}=20$ & $\operatorname{Pr}=25$ \\
\hline 0.0 & \multirow{6}{*}{1.0} & 0.7145 & 0.8098 & 1.0370 & 1.1239 \\
\hline 0.5 & & 0.7104 & 0.8063 & 1.0322 & 1.1190 \\
\hline 1.0 & & 0.7072 & 0.8028 & 1.0289 & 1.1158 \\
\hline 2.0 & & 0.7012 & 0.7966 & 1.0226 & 1.1095 \\
\hline 3.0 & & 0.6961 & 0.7912 & 1.0168 & 1.1036 \\
\hline 4.0 & & 0.6917 & 0.7865 & 1.0117 & 1.0986 \\
\hline \multirow{6}{*}{0.3} & 0.0 & 0.8013 & 0.9019 & 1.1364 & 1.2267 \\
\hline & 0.5 & 0.7497 & 0.8463 & 1.0747 & 1.1628 \\
\hline & 1.0 & 0.7125 & 0.8089 & 1.0330 & 1.1213 \\
\hline & 2.0 & 0.6568 & 0.7512 & 0.9736 & 1.0593 \\
\hline & 3.0 & 0.6144 & 0.7072 & 0.9277 & 1.0122 \\
\hline & 4.0 & 0.5799 & 0.6710 & 0.8902 & 0.9735 \\
\hline
\end{tabular}

Table 8: Values of $f^{\prime \prime}(\xi, 0)$ for different values of $W e$, $M$ and $P r$

\begin{tabular}{|c|c|c|c|c|}
\hline \multirow{2}{*}{$W e$} & \multirow{2}{*}{$M$} & \multicolumn{3}{|c|}{$f^{\prime \prime}(\xi, 0)$} \\
\hline & & $\operatorname{Pr}=50$ & $\operatorname{Pr}=75$ & $\operatorname{Pr}=100$ \\
\hline 0.0 & \multirow{6}{*}{1.0} & 0.1749 & 0.1524 & 0.1370 \\
\hline 0.5 & & 0.1762 & 0.1533 & 0.1377 \\
\hline 1.0 & & 0.1771 & 0.1541 & 0.1383 \\
\hline 2.0 & & 0.1795 & 0.1557 & 0.1393 \\
\hline 3.0 & & 0.1815 & 0.1571 & 0.1407 \\
\hline 4.0 & & 0.1833 & 0.1584 & 0.1417 \\
\hline \multirow{6}{*}{0.3} & 0.0 & 0.2194 & 0.1924 & 0.1745 \\
\hline & 0.5 & 0.1923 & 0.1677 & 0.1512 \\
\hline & 1.0 & 0.1757 & 0.1529 & 0.1374 \\
\hline & 2.0 & 0.1517 & 0.1315 & 0.1175 \\
\hline & 3.0 & 0.1332 & 0.1149 & 0.1022 \\
\hline & 4.0 & 0.1177 & 0.1011 & 0.0893 \\
\hline
\end{tabular}

Table 9: Values of $-\theta^{\prime}(\xi, 0)$ for different values of $W e, M$ and $P r$

\begin{tabular}{|c|c|c|c|c|}
\hline \multirow{2}{*}{$W e$} & \multirow{2}{*}{$M$} & \multicolumn{3}{|c|}{$-\theta^{\prime}(\xi, 0)$} \\
\hline & & $\mathrm{Pr}=50$ & $\operatorname{Pr}=75$ & $\operatorname{Pr}=100$ \\
\hline 0.0 & \multirow{6}{*}{1.0} & 1.4513 & 1.6946 & 1.8975 \\
\hline 0.5 & & 1.4473 & 1.6911 & 1.8942 \\
\hline 1.0 & & 1.4430 & 1.6879 & 1.8913 \\
\hline 2.0 & & 1.4376 & 1.6821 & 1.8846 \\
\hline 3.0 & & 1.4321 & 1.6769 & 1.8812 \\
\hline 4.0 & & 1.4272 & 1.6724 & 1.8770 \\
\hline \multirow{6}{*}{0.3} & 0.0 & 1.5630 & 1.8118 & 2.0183 \\
\hline & 0.5 & 1.4925 & 1.7373 & 1.9413 \\
\hline & 1.0 & 1.4489 & 1.6925 & 1.8955 \\
\hline & 2.0 & 1.3850 & 1.6270 & 1.8289 \\
\hline & 3.0 & 1.3355 & 1.5762 & 1.7772 \\
\hline & 4.0 & 1.2928 & 1.5333 & 1.7334 \\
\hline
\end{tabular}




\section{CONCLUSIONS}

A theoretical study has been conducted of laminar incompressible free convection boundary layer flow of a viscoelastic (Williamson) fluid from a vertical truncated cylinder. Magnetic field, Momentum and thermal slip effects have been incorporated in the model. The transformed boundary layer equations for heat and momentum conservation have been solved using a finite difference method for the case of non-similar solutions present at the cylinder surface. Verification of the accuracy of the Keller box computational code has been achieved via comparison with previous Newtonian solutions reported in the literature. The present investigation has shown that:

1) Increasing the velocity slip parameter $(S f)$ reduces the velocity near the cylinder surface and increases the temperature i.e. enhances momentum boundary layer thickness and decreases thermal boundary layer thickness. However, flow reversal is never computed.

2) Increasing thermal slip parameter, $\left(S_{T}\right)$ consistently decelerates the flow and also decreases temperature (and thermal boundary layer thickness).

3) Increasing Williamson viscoelastic fluid parameter ( $W e$ ), decreases the velocity near the surface and also fractionally lowers the temperature throughout the boundary layer regime i.e. enhances thermal boundary layer thickness.

4) Increasing Prandtl number $(P r)$ decelerates the flow and also strongly depresses temperatures, throughout the boundary layer regime.

\section{NOMENCLATURE}

$\mathrm{B}_{0} \quad$ externally imposed radial magnetic field

$\mathrm{C}_{\mathrm{f}} \quad$ skin friction coefficient

f non-dimensional steam function

Gr Grashof number

g acceleration due to gravity

$\mathrm{k}$ thermal conductivity of fluid

$\mathrm{K}_{0} \quad$ thermal jump factor

$\mathrm{Nu} \quad$ local Nusselt number

M magnetic body force parameter

Pr Prandtl number

No velocity (momentum) slip factor

$\mathrm{S}_{\mathrm{f}} \quad$ non-dimensional velocity slip parameter

$\mathrm{S}_{\mathrm{T}} \quad$ non-dimensional thermal jump parameter

$\mathrm{T}$ temperature

$\mathrm{u}, \mathrm{v} \quad$ non-dimensional velocity components along the $\mathrm{x}-$ and $\mathrm{y}$ directions, respectively

We Weissenberg (viscoelasticity) number

$\mathrm{x} \quad$ stream wise coordinate

y transverse coordinate

\section{Greek Symbols}

$\alpha \quad$ thermal diffusivity

$\beta \quad$ coefficient of thermal expansion

$\eta \quad$ dimensionless transverse coordinate

$v \quad$ kinematic viscosity

$\theta \quad$ non-dimensional temperature

$\rho \quad$ density of viscoelastic fluid

$\sigma \quad$ electrical conductivity of viscoelastic fluid

$\xi \quad$ dimensionless steam wise coordinate

$\psi \quad$ dimensionless stream function

$\Gamma \quad$ time-dependent material constant

\section{Subscripts}

w conditions on the wall

$\infty \quad$ Free stream conditions

\section{Acknowledgment}

The corresponding author is thankful to Dr. Abderrahim Wakif and Dr. Zoubair Boulahia, Hassan II University Faculty of Sciences, Mechanical Laboratory Group of Simulation and Optimization in Heat and Mass Transfer for the Nanofluids Casablanca, Morocco.

\section{REFERENCES}

Afonso, A.M., Ferrás, L. L., Nóbrega, J. M., Alves, M.A., Pinho, F. T., 2014, "Pressure-Driven Electrokinetic Slip Flows of Viscoelastic Fluids in Hydrophobic Microchannels," Microfluidics and Nanofluidics, 16(6), 1131-1142.

https://doi.org/10.1007/s10404-013-1279-5

Aly, A.A., 2015, "Heat Treatment of Polymers: A Review," Int. J. Materials Chemistry and Physics, 1(2), 132-140.

Aldoss, T.K., Ali, Y.D., and A-Nimr, M.A., 1996, "MHD Mixed Convection from A Horizontal Circular Cylinder," Numerical heat transfer part A, 30, 379-396.

Alireza, R., Davood, D., and Ganji, S.T., 2012, "Numerical Solutions for A Nanofluid Past Over a Stretching Circular Cylinder with NonUniform Heat Source," Frontiers in Heat and Mass Transfer, 3 043003 .

Amanulla, C.H., Nagendra, N., and Suryanarayana Reddy, M., 2018, "Numerical Simulations on Magnetohydrodynamic Non-Newtonian Nanofluid Flow Over a Semi-Infinite Vertical Surface with Slip effects," Journal of Nanofluids, 7(4), 718-730.

https://doi.org/10.1166/jon.2018.1499

Amanulla, C.H., Nagendra, N., and Suryanarayana Reddy, M., 2017, "Multiple Slip Effects on MHD and Heat Transfer in a Jeffery Fluid over an Inclined Vertical Plate," International Journal of Pure and Applied Mathematics, 113(7), 137-145.

Amanulla, C.H., Nagendra, N., Subba Rao A., Anwar Bég O., and Kadir A., 2018, "Numerical Exploration of Thermal Radiation and Biot Number Effects on the Flow of a Non-Newtonian MHD Williamson Fluid over a Vertical Convective Surface," Heat Trans Asian Res., 47 (2), 286-304.

https://doi.org/10.1002/htj.21303

Amanulla, C.H., Nagendra, N., Surya Narayana Reddy, M., Subba Rao, A., and Anwar Bég, O., 2017, "Mathematical Study of Non-Newtonian Nanofluid Transport Phenomena from an Isothermal Sphere," Frontiers in Heat and Mass Transfer, 8, 29.

http://dx.doi.org/10.5098/hmt.8.29

Amanulla, C.H., Nagendra, N., and Surya Narayana Reddy, M., 2017, "Numerical Study of Thermal and Momentum Slip Effects on MHD Williamson Nanofluid from an Isothermal Sphere," Journal of Nanofluids, 6(6), 1111-1126.

https://doi.org/10.1166/jon.2017.1405

Amanulla, C.H., Nagendra, N., and Suryanarayana Reddy, M., 2017, "MHD Flow and Heat Transfer in a Williamson Fluid from a Vertical Permeable Cone with Thermal and Momentum Slip Effects: A Mathematical Study," Frontiers in Heat and Mass Transfer, 8, 40. http://dx.doi.org/10.5098/hmt.8.40

Amanulla, C.H., Nagendra, N., and Suryanarayana Reddy, M., 2017, "Computational Analysis of Non-Newtonian Boundary Layer Flow of Nanofluid Past a Semi-infinite Vertical Plate with Partial Slip," Nonlinear Engineering, 1-15.

https://doi.org/10.1515/nleng-2017-0055 
Amanulla, C.H., Nagendra, N., and Suryanarayana Reddy, M., 2017, "Thermal and Momentum Slip Effects on Hydromagnetic Convection Flow of a Williamson Fluid Past a Vertical Truncated Cone," Frontiers in Heat and Mass Transfer, 9, 22.

https://doi.org/10.5809/hmt.9.22

Black, W.B., 2000, "Wall slip and boundary effects in polymer shear flows," PhD Thesis, Chemical Engineering, University of Wisconsin Madison, USA.

Cebeci, T., and Bradshaw, P., 1984, "Physical and Computational Aspects of Convective Heat Transfer," Springer. New York.

Chiu-On Ng, 2017, "Starting flow in Channels with Boundary Slip," Meccanica, 52(1-2), 45-67.

https://doi.org/10.1007/s11012-016-0384-4

Dapra, I., and Scarpi, G., 2007, "Perturbation Solution for Pulsatile Flow of a Non-Newtonian Williamson Fluid in a Rock Fracture," Int. J. Rock Mechanics and Mining Sciences, 44(2), 271-278.

Dasman, A., 2010, "Mixed Convection Boundary Layer Flow of a Viscoelastic Fluid Past a Sphere", Masters thesis, Universiti Teknologi Malaysia, Faculty of Science.

Dealy, J., and Wissbrun, K., 1990, "Melt Rheology and its Role in Plastics Processing: Theory and Applications", Van Nostrand Reinhold, New York.

Ferrás, L.L., Nóbrega, J.M., and Pinho, F.T., 2013, "Implementation of Slip Boundary Conditions in The Finite Volume Method: New Techniques," Int. J. Numer. Meth. Fluids, 72(7), 724-747. https://doi.org/10.1002/fld.3765

Ferrás, L.L., Nóbrega, J.M., and Pinho, F.T., 2012, “Analytical Solutions for Channel Flows of Phan-Thien-Tanner and Giesekus Fluids Under Slip," J. Non-Newtonian Fluid Mech., 171-172, 97-105. https://doi.org/10.1016/j.jnnfm.2012.01.009

Ferrás, L.L., Afonso, A.M., Alves, M.A., Nóbrega, J.M., Carneiro, O.S., and Pinho, F.T., 2014, "Slip Flows of Newtonian and Viscoelastic Fluids in a 4:1 Contraction," J. Non-Newtonian Fluid Mech., 214, 2837.

https://doi.org/10.1016/j.jnnfm.2014.09.007

Ferrás, L.L., Afonso, A.M., Alves, M.A., Nóbrega, J.M., and Pinho, F. T., 2014, "Annular Flow of Viscoelastic Fluids: Analytical and Numerical Solutions," J. Non-Newtonian Fluid Mech., 212, 80-91. https://doi.org/10.1016/j.jnnfm.2014.07.004

Ferrás, L.L., Afonso, A.M., Nóbrega, J.M., and Pinho, F.T., 2017, “A Numerical and Theoretical Study on Viscoelastic Fluid Slip Flows," Physics of Fluids, 29, 053102.

http://dx.doi.org/10.1063/1.4983062

George, K., and Georgiou, G.C., 2015, "Start-Up and Cessation Newtonian Poiseuille and Couette Flows with Dynamic Wall Slip," Meccanica, 50(7), 1747-1760.

Ghannam, M., and Esmail N., 2002, "Flow Behavior of Enhanced Oil Recovery Alcoflood Polymers", J. Applied Polymer Science, 85(14), $2896-2904$

Grigoriadis, D.G.E., Sarris, I.E., and Kassinos, S.C., 2010, "MHD Flow Past a Circular Cylinder Using the Immersed Boundary Method," Computers \& Fluids, 39, 345-358.

http://dx.doi.org/10.1016/j.compfluid.2009.09.012
Hayat, T., Shafiq, A., and Alsaedi, A., 2016, "Hydromagnetic Boundary Layer Flow of Williamson Fluid in The Presence of Thermal Radiation and Ohmic Dissipation," Alexandria Engineering J., 55(3), 2229-2240. http://dx.doi.org/10.1016/j.aej.2016.06.004

Hayat, T., Hussain, Z., Alsaedi, A., and Farooq, M., 2016 "Magnetohydrodynamic Flow by a Stretching Cylinder with Newtonian Heating and Homogeneous-Heterogeneous Reactions," PLoS ONE 11(6).

http://dx.doi.org/10.1371/journal.pone.0156955

Hayat T., Shafiq A., Alsaedi A., and Shahzad S. A.,2016, "Unsteady MHD Flow Over Exponentially Stretching Sheet with Slip Conditions," Applied Mathematics and Mechanics, 37(2),193-208.

https://doi.org/10.1007/s10483-016-2024-8

Hatzikiriakos, S.G., and Kalogerakis, N., 1994, "A Dynamic Slip Velocity Model for Molten Polymers Based on a Network Kinetic Theory," Rheol. Acta, 33, 38-47.

https://doi.org/10.1007/BF00453462

Hatzikiriakos, S.G., and Mitsoulis, E., 2009, "Slip Effects in Tapered Dies", Polymer Engineering and Science, 49(10),1960 - 1969.

https://doi.org/10.1002/pen.21430

Hunegnaw, D., and Naikoti, K., 2014, "MHD Effects on Heat Transfer Over Stretching Sheet Embedded in Porous Medium with Variable Viscosity, Viscous Dissipation and Heat Source/Sink," Ain Shams Engineering Journal,5(3), 967-977. https://doi.org/10.1016/j.asej.2014.03.008

Javed T., Majeed, A., and Mustafa, I., 2015, "MHD Effects On Natural Convection Laminar Flow from a Horizontal Circular Cylinder in Presence of Radiation," Revista Mexicana de Fisica, 61, 450-457.

Kasim, A.R.M., Mohammad, N.F., Anwar, I., and Shafie, S., 2013, "MHD Effect On Convective Boundary Layer Flow of a Viscoelastic Fluid Embedded in Porous Medium with Newtonian Heating," Recent Advances in Mathematics, 4, 182-189.

Keller, H.B., 1970, "A New Difference Method for Parabolic Problems. Numerical Methods for Partial Differential Equations," J. Bramble (Editor). Academic Press. New York. USA.

Khan, N.A., and Khan, H., 2014, "A Boundary Layer Flows of NonNewtonian Williamson Fluid," Nonlinear Engineering, 3(2), 107-115. http://dx.doi.org/10.1515/nleng-2014-0002

Lim, F.J., and Schowalter, W.R., 1998, "Wall Slip of Narrow Molecular Weight Distribution Polybutadienes," J. Rheol., 33(8), 1359-1382. http://dx.doi.org/10.1122/1.550073

Liu, Y., and Gehde, M., 2016, "Effects of Surface Roughness and Processing Parameters on Heat Transfer Coefficient Between Polymer and Cavity Wall During Injection Molding," The Int. J. Advanced Manufacturing Technology, 84, 1325-1333.

https://doi.org/10.1007/s00170-015-7816-5

Loix, F., Orgéas, L., Geindreau, C., Badel, P., Boisse, P., and Bloch, J.F., 2009, "Flow of Non- Newtonian Liquid Polymers Through Deformed Composites Reinforcements," Composites Science and Technology, 69, 612-619.

Megahed, A.M., 2012, "Variable Viscosity and Slip Velocity Effects on The Flow and Heat Transfer of a Power-Law Fluid Over a NonLinearly Stretching Surface with Heat Flux and Thermal Radiation," Rheologica Acta, 51(9), 841-847. http://dx.doi.org/10.1007/s00397-012-0644-8 
Makanda, G., Shaw, S., and Sibanda, P., 2015, "Effects of Radiation on MHD Free Convection of a Casson Fluid from a Horizontal Circular Cylinder with Partial Slip in Non-Darcy Porous Medium with Viscous Dissipation," Boundary Value Problems, 75-84. http://dx.doi.org/10.1186/s13661-015-0333-5

Merkin J.H., 1976, "Free Convection Boundary Layer on an Isothermal Horizontal Circular Cylinders," ASME/AIChE, Heat Transfer Conference, St. Louis, Mo., U.S.A. 9-11.

Molla, M.M., Saha, S.C., Khan, M.A.I., and Hossain, M.A., 2011, "Radiation Effects on Natural Convection Laminar Flow from a Horizontal Circular Cylinder," Desalin. Water Treat., 30, 89-97. https://doi.org/10.5004/dwt.2011.1870

Panaseti, P., and Georgiou, G.C., 2017, "Viscoplastic Flow Development in a Channel with Slip Along One Wall," J. NonNewtonian Fluid Mech., 248, 8-22.

https://doi.org/10.1016/j.jnnfm.2017.08.008

Piau, J.M., Kissi, N.E., Toussaint, F., and Mezghani, A., 1995, "Distortions of Polymer Extrudates and Their Elimination Using Slippery Surfaces," Rheol. Acta, 34(1), 40-57. http://dx.doi.org/10.1007/BF00396053

Philippou, M., Damianou, Y., Xenia, M., and Georgiou, G.C., 2017, "Cessation of Newtonian Circular and Plane Couette Flows with Wall Slip and Non-Zero Slip Yield Stress," Meccanica, 52, 2081-2099. https://doi.org/10.1007/s11012-016-0565-1

Piau, J.M., and Kissi, N.E., 1994, "Measurement and Modelling of Friction in Polymer Melts During Macroscopic Slip at The Wall," $J$. Non-Newtonian Fluid Mech., 54, 121-142.

http://dx.doi.org/10.1016/0377-0257(94)80018-9

Prasannakumara, B.C., Gireesha, B.J., Gorla, R.S.R., and Krishnamurthy, M.R., 2016, "Effects of Chemical Reaction and Nonlinear Thermal Radiation on Williamson Nanofluid Slip Flow Over a Stretching Sheet Embedded in A Porous Medium," J. Aerosp. Eng., 29(5).

http://dx.doi.org/10.1061/(ASCE)AS.1943-5525.0000578

Rao, K.S., and Rao, P.K., 2014, "Fully Developed Free Convective Flow of a Williamson Fluid Through a Porous Medium in a Vertical Channel," Int. J. Conceptions on Computing and Information Technology, 2(1), 54-57.

Rao, S.A., Amanulla, C.H., Nagendra, N., Surya Narayana Reddy, M., and Bég, O. A., 2018, "Hydromagnetic Non-Newtonian Nanofluid Transport Phenomena Past an Isothermal Vertical Cone with Partial Slip: Aerospace Nanomaterial Enrobing Simulation," Heat Trans Asian Res., 47(1), 203-230. http://dx.doi.org/10.1002/htj.21299.

Rao, C.V.R., Sekhar, T.V.S., 2000, "MHD Flow Past a Circular Cylinder-A Numerical Study," Computational Mechanics, 26(5),430436.

http://dx.doi.org/10.1007/s004660000191
Salahuddin, T., Malik M. Y., Hussain, A., Bilal, S., and Awais M., 2016, "Combined Effects of Variable Thermal Conductivity and MHD Flow on Pseudoplastic Fluid over a Stretching Cylinder by using Keller Box Method," Information Sciences Letters,5(1), 11-19 http://dx.doi.org/10.18576/is1/050102

Salleh, M. Z., Nazar, R., Arifin, N. M., Pop, I., and Merkin, J. H., 2011, "Forced-Convection Heat Transfer Over a Circular Cylinder with Newtonian Heating," J. Eng. Math., 69, 101-110.

https://doi.org/10.1007/s10665-010-9408-6

Saleem, S., and Nadeem, S., 2015, "Theoretical Analysis of Slip Flow on a Rotating Cone with Viscous Dissipation Effects," $J$. Hydrodynamics. 27(6), 616-623.

https://doi.org/10.1016/S1001-6058(15)60523-6

Saleem, S., Nadeem, S. and Awais, M., 2016, “Time Dependent Second Order Viscoelastic Fluid Flow on a Rotating Cone with Heat Generation and Chemical Reaction," J. Aerospace Eng., 29(4). https://doi.org/10.1061/(ASCE)AS.1943-5525.0000599

Swati, M., and Anuar, I., 2012, "Mixed Convection Flow along a Stretching Cylinder in a Thermally Stratified Medium," J. App. Math., 2012(2012).

http://dx.doi.org/10.1155/2012/491695

Sato, S., Oka, K., and Murakami, A., 2004, "Heat Transfer Behavior of Melting Polymers in Laminar Flow Field," Polymer Eng., Sci., 44(3), 423-432.

http://dx.doi.org/10.1002/pen.20038

Tapas Ray, M., Sumanta, S., and Samir Kumar, N., 2014, "Effect of Magnetic Field on Indirect Natural Convection Flow Above a Horizontal Hot Flat Plate," Frontiers in Heat and Mass Transfer, 5, 15. http://dx.doi.org/10.5098/hmt.5.15

Yamaguchi, H., Zhang, X.R., Higashi, S., and Li, M., 2008, "Study on Power Generation Using Electro-Conductive Polymer and Its Mixture with Magnetic Fluid," J. Magnetism and Magnetic Materials, 320(7), 1406-1411.

http://dx.doi.org/10.1016/j.jmmm.2007.12.014

Wang, S.Q., Drda, P.A., and Inn, Y.W., 1996, "Exploring Molecular Origins of Sharkskin, Partial Slip, And Slope Change in Flow Curves of Linear Low Density Polyethylene," J. Rheology, 40(5), 875- 898. https://doi.org/10.1122/1.550766

Williamson, R.V., 1929, "The Flow of Pseudoplastic Materials," Industrial and Engineering Chemistry, 21(11), 1108-1111.

https://doi.org/10.1021/ie50239a035

Wubshet, I., Bandari, S., 2016, "The Effects of Thermal Radiation and Non-Uniform Heat Source/Sink on MHD Boundary-Layer Flow and Heat Transfer Past a Stretching Sheet Embedded in Non-Darcian Porous Medium," Frontiers in Heat and Mass Transfer, 7, 37. http://dx.doi.org/10.5098/hmt.7.37 